\title{
The existence of positive solutions for Kirchhoff-type problems via the sub-supersolution method
}

\author{
Baoqiang Yan, Donal O'Regan and Ravi P. Agarwal
}

\begin{abstract}
In this paper we discuss the existence of a solution between wellordered subsolution and supersolution of the Kirchhoff equation. Using the sub-supersolution method together with a Rabinowitz-type global bifurcation theory, we establish the existence of positive solutions for Kirchhoff-type problems when the nonlinearity is singular or sign-changing. Moreover, we obtain some necessary and sufficient conditions for the existence of positive solutions for the problem when $N=1$.
\end{abstract}

\section{Introduction}

In this paper, we consider the following nonlocal elliptic problem

$$
\left\{\begin{array}{l}
-a\left(\int_{\Omega}|\nabla u(x)|^{2} d x\right) \Delta u(x)=f(x, u), \quad x \text { in } \Omega \\
u>0, \quad x \text { in } \Omega \\
u=0, \quad x \text { on } \partial \Omega
\end{array}\right.
$$

where $\Omega \subseteq R^{N}$ is a smooth bounded domain. This problem is related to the stationary analogue of the Kirchhoff equation $\left(a(t)=a_{1}+a_{2} t, a_{1}>0\right.$,

Key Words: Kirchhoff-type elliptic problems; Rabinowitz-type global bifurcation theory; Sub-supersolution method; Existence; Uniqueness.

2010 Mathematics Subject Classification: 35J60, 35J75, 47H10.

This research is supported by the Fund of Science and Technology Plan of Shandong Province (2014GGH201010) and the NSFC(61603226).

Received: 20.12.2016

Revised: 10.09.2017

Accepted: 20.09.2017 
$a_{2}>0$ ) which was proposed by Kirchhoff as a generalization of the well-known d'Alembert's equation

$$
\rho \frac{\partial^{2} u}{\partial t^{2}}-\left(\frac{P_{0}}{h}+\frac{E}{2 L} \int_{0}^{L}\left|\frac{\partial u}{\partial x}\right|^{2} d x\right) \frac{\partial^{2} u}{\partial x^{2}}=g(x, u)
$$

for free vibrations of elastic strings; see [21]. Kirchhoff's model takes into account the changes in length of the string produced by transverse vibrations, in which $L$ is the length of the string, $h$ is the area of the cross section, $E$ is the Young modulus of the material, $\rho$ is the mass density and $P_{0}$ is the initial tension. Problem (1.1) received some attention after the paper by Lions [29], where an abstract framework to the problem was proposed and variational methods were applied to establish existence and multiplicity of positive solutions for problem (1.1) when $f$ is continuous at $u=0$; see also $[3-4,6,12,18-20,24,27,32-33,35]$ and the references therein. There are only a few results on the existence of positive solutions to problem (1.1) when $f$ is singular at $u=0$. When $f$ includes functions like $1 / u^{-\mu}, \mu \in(0,1)$, Liu and Sun in [30], Lei el. in [23] and Liao el. in [28] considered multiplicity (using variational methods) of positive solutions for problem (1.1).

The sub-supersolution method is an important tool to establish the existence of solutions to an elliptic problem like (1.1); see [7, 9, 17, 31]. However the presence of a nonlocal term leads to the some additional conditions: (1) the nonlinearity $f$ is nondecreasing; or $(2) a(t)$ is bounded. Two recent papers [14-16] pointed out some errors in the literatures and the authors obtained some theorems using a sub-supersolution method.

There are two main objectives in this paper. First from the ideas in $[2$, $6-7,9,11,14-15,17,25-26,31,37-38]$, we present some new definitions of sub-supersolutions to problem (1.1) and we obtain the existence of classical solutions to problem (1.1) between subsolution and supersolution. Second we present conditions for the existence of positive solutions to problem (1.1) when $f$ is singular at $u=0$ or $f$ is sign-changing.

The paper is organized as follows. In Section 2, we prove some new results on the existence of classical solutions between subsolution and supersolution using the maximum principle and in Section 3, existence and uniqueness results of positive solution for (3.1) are presented. In Section 4, we discuss the existence of positive solutions to problems (4.1) and (4.2) and the asymptotic behavior of positive solutions for large $\lambda$. In Section 5, we present necessary and sufficient conditions on the existence of positive solutions for problems (5.1) when $N=1$. 


\section{Sub-supersolution method}

Now we consider a general problem

$$
\left\{\begin{array}{l}
-a\left(\int_{\Omega}|\nabla u(x)|^{2} d x\right) \Delta u(x)=F(x, u(x)), \quad x \text { in } \Omega \\
u=0, \quad x \text { on } \partial \Omega
\end{array}\right.
$$

where $\Omega \subseteq R^{N}$ is a smooth bounded domain, and $a:[0,+\infty) \rightarrow(0,+\infty)$ is a continuous and nondecreasing function with

$$
\inf _{t \in[0,+\infty)} a(t) \geq a(0) \stackrel{\text { def. }}{=} a_{0}>0 .
$$

Let $C^{1}(\bar{\Omega})=\{u: \bar{\Omega} \rightarrow R \mid u(x)$ is continuously differentiable on $\bar{\Omega}\}$ with norm $\|u\|=\max \left\{\max _{x \in \bar{\Omega}}|u(x)|, \max _{x \in \bar{\Omega}}|\nabla u(x)|\right\}$. It is easy to see that $C^{1}(\bar{\Omega})$ is a Banach space.

Definition 2.1. The pair functions $\alpha$ and $\beta$ with $\alpha, \beta \in C^{1}(\bar{\Omega}) \cap C^{2}(\Omega)$ are subsolution and supersolution of (2.1) if $\alpha(x) \leq u(x) \leq \beta(x)$ for $x \in \Omega$, and

$$
\begin{aligned}
& \left\{\begin{array}{l}
-\Delta \alpha(x) \leq \frac{1}{b_{0}} F(x, \alpha(x)), \quad x \text { in } \Omega, \\
\left.\alpha\right|_{\partial \Omega} \leq 0,
\end{array}\right. \\
& \left\{\begin{array}{l}
-\Delta \beta(x) \geq \frac{1}{a_{0}} F(x, \beta(x)), \quad x \text { in } \Omega, \\
\left.\beta\right|_{\partial \Omega} \geq 0,
\end{array}\right.
\end{aligned}
$$

where $a_{0}=a(0)$ and $b_{0}=a\left(\int_{\Omega} H^{2}(x) d x\right), E \in L^{p}(\Omega)(p>N)$; here

$$
\begin{gathered}
E(x)=\sup _{u \in[\alpha(x), \beta(x)]}|F(x, u)|, \quad x \in \bar{\Omega}, \\
H(x)=\frac{1}{a_{0}} \int_{\Omega}\left|G_{x}(x, y)\right| E(y) d y, \quad x \in \bar{\Omega},
\end{gathered}
$$

and $G(x, y)$ is the Green's function for $-\Delta u(x)=h$ and $\left.u\right|_{\partial \Omega}=0$.

From the ideas in [11], we give the following definitions.

Definition 2.2. Let $u, v \in C^{1}(\bar{\Omega})$. We say that $u \prec v$ if $u(x)<v(x)$ on $\Omega$ and $u(x) \leq v(x)$ for all $x \in \partial \Omega$ and if $u(x)=v(x)$ for some $x \in \Gamma \subseteq \partial \Omega$, $\left.\frac{\partial u}{\partial n}\right|_{x \in \partial \Gamma}>\left.\frac{\partial \bar{v}}{\partial n}\right|_{x \in \Gamma}$.

Remark 2.1. $S=\left\{u \in C^{1}(\bar{\Omega}): \alpha \prec u \prec \beta\right\}$ is an open set if $\alpha \prec \beta$.

We say that an open set $S \subseteq C^{1}(\bar{\Omega})$ is admissible for the degree (for the compact map $A$ ) if the compact operator $A$ has no fixed point on its boundary $\partial S$ and the set of fixed points of $A$ in $S$ is bounded. 
In that case, we define

$$
\operatorname{deg}(I-A, S, \theta)=\operatorname{deg}(I-A, S \cap B(0, R), \theta)
$$

where $R$ is such that every fixed point $u$ of $A$ in $S$ satisfies $\|u\|<R$. From the excision property this degree does not depend on $R$.

To be able to associate a degree to a pair of subsolution and supersolution we have to reinforce the definition.

Definition 2.3. A subsolution $\alpha$ of (2.1) is said to be strict if every solution $u$ of (2.1) such that $\alpha \leq u$ satisfies $\alpha \prec u$.

In the same way a strict supersolution $\beta$ of (2.1) is a supersolution such that every solution $u$ of (2.1) such that $u \leq \beta$ satisfies $u \prec \beta$.

Definition 2.4. The function $F: \Omega \times R$ is an $L^{p}$-Caratheodory function if

1. $F(\cdot, u)$ is measurable for all $u \in \Omega$;

2. $F(x, \cdot)$ is continuous for a.e. $x \in \Omega$;

3. for all bounded set $B \subseteq R^{N}$, there exists $h_{B} \in L^{p}(\Omega)$ such that for a.e. $x \in \Omega$ and all $u \in B$,

$$
|F(x, u)| \leq h_{B}(x) .
$$

Remark 2.2. The idea for the above definitions came from $[2,11]$.

If $F$ is an $L^{p}$-Caratheodory function and $(\alpha, \beta)$ are subsolution and supersolution to (2.1) as in Definition 2.1, then the operator

$$
N: C^{1}(\bar{\Omega}) \rightarrow L^{p}(\Omega): u \mapsto \frac{F(x, u(x))}{a\left(\int_{\Omega}|| \nabla u(x)\left|-(|\nabla u(x)|-H(x))^{+}\right|^{2} d x\right)}
$$

is well-defined, continuous, and maps bounded sets to bounded sets; here $(|\nabla u(x)|-H(x))^{+}=\max \{0,|\nabla u(x)|-H(x)\}$. Then the operator $A: C^{1}(\bar{\Omega}) \rightarrow$ $C^{1}(\bar{\Omega})$

$$
A u=(-\Delta)^{-1}(N u)
$$

is completely continuous.

Theorem 2.1. Let $\Omega \subseteq R^{N}(N \geq 1)$ be a smooth bounded domain. Suppose that $F: \Omega \times R \rightarrow R$ is a continuous function. Assume $\alpha$ and $\beta$ are the subsolution and supersolution of (2.1) respectively. If

$$
F(x, u) \geq 0, x \in \Omega, \alpha(x) \leq u \leq \beta(x),
$$

then problem (2.1) has at least one solution $u$ such that, for all $x \in \bar{\Omega}$,

$$
\alpha(x) \leq u(x) \leq \beta(x) .
$$


If moreover $\alpha(x)$ and $\beta(x)$ are strict and satisfy $\alpha \prec \beta$, then

$$
S=\left\{u \in C^{1}(\bar{\Omega}) \mid \alpha \prec \beta\right\} .
$$

is admissible for the degree (for the map $A$ ) and

$$
\operatorname{deg}(I-A, S, \theta)=1 \text {. }
$$

Proof. Let

$$
\bar{F}(x, u)=\left\{\begin{array}{l}
F(x, \alpha(x)), \text { if } u<\alpha(x) \\
F(x, u), \text { if } \alpha(x) \leq u \leq \beta(x) \\
F(x, \beta(x)), \text { if } u>\beta(x)
\end{array}\right.
$$

We will study the modified problem

$$
\left\{\begin{array}{l}
-\Delta u=\frac{\bar{F}(x, u)}{a\left(\int_{\Omega}|| \nabla u(x)\left|-(|\nabla u(x)|-H(x))^{+}\right|^{2} d x\right)}, x \in \Omega, \\
\left.u\right|_{\partial \Omega}=0 .
\end{array}\right.
$$

Step 1. Every solution $u$ of (2.3) satisfies $\alpha(x) \leq u(x) \leq \beta(x), x \in \bar{\Omega}$.

We prove that $\alpha(x) \leq u(x)$ on $\bar{\Omega}$. Obviously, ||$\nabla u(x) \mid-(|\nabla u(x)|-$ $H(x))\left.^{+}\right|^{2} \leq H(x)^{2}$, which together with the monotonicity of $a(t)$ implies that

$$
a_{0} \leq a\left(\int_{\Omega}|| \nabla u(x)\left|-(|\nabla u(x)|-H(x))^{+}\right|^{2} d x\right) \leq a\left(\int_{\Omega} H(x)^{2} d x\right) .
$$

By contradiction, assume that $\max _{x \in \bar{\Omega}}(\alpha(x)-u(x))=M>0$. Note that $\alpha(x)-u(x) \not \equiv M$ on $\bar{\Omega}(\alpha(x)-u(x) \leq 0, x \in \partial \Omega)$. If $x_{0} \in \Omega$ is such that $\alpha\left(x_{0}\right)-u\left(x_{0}\right)=M$, choose $A_{0}=\{x \in \Omega \mid \alpha(x)-u(x)>0\}$ a connected domain with $x_{0} \in A_{0}$. It follows from (2.2) that

$$
\begin{aligned}
& -\Delta(\alpha(x)-u(x)) \\
& \leq \frac{1}{b_{0}} F(x, \alpha(x))-\frac{1}{a\left(\int_{\Omega}|| \nabla u(x)\left|-(|\nabla u(x)|-H(x))^{+}\right|{ }^{2} d x\right)} \bar{F}(x, u(x)) \\
& \leq \frac{1}{b_{0}}(F(x, \alpha(x))-F(x, \alpha(x))) \\
& \leq 0, \quad x \in A_{0}
\end{aligned}
$$

and

$$
\alpha(x)-u(x)=0, \quad x \in \partial A_{0} .
$$

By the maximum principle, one has $\alpha(x)-u(x) \leq 0$ for $x \in A_{0}$. This contra$\operatorname{dicts} \alpha\left(x_{0}\right)-u\left(x_{0}\right)>0$.

Now we prove that $\beta(x) \geq u(x)$ on $\bar{\Omega}$. By contradiction, assume that $\min _{x \in \bar{\Omega}}(\beta(x)-u(x))=-m<0$. Note that $\beta(x)-u(x) \not \equiv-m$ on $\bar{\Omega}(\beta(x)-$ 
$u(x) \geq 0, x \in \partial \Omega)$. If $x_{0} \in \Omega$ is such that $\beta\left(x_{0}\right)-u\left(x_{0}\right)=-m$, choose $B_{0}=\{x \in \Omega \mid \beta(x)-u(x)<0\}$ a connected domain with $x_{0} \in B_{0}$. It follows from (2.2) that

$$
\begin{aligned}
& -\Delta(\beta(x)-u(x)) \\
& \geq \frac{1}{a_{0}} F(x, \beta(x))-\frac{1}{\left.a\left(\int_{\Omega}|| \nabla u(x)\left|-(|\nabla u(x)|-H(x))^{+}\right|^{2} d x\right)\right)} \bar{F}(x, u(x)) \\
& \geq \frac{1}{a_{0}}(F(x, \beta(x))-F(x, \beta(x))) \\
& \geq 0, \quad x \in B_{0}
\end{aligned}
$$

and

$$
\beta(x)-u(x)=0, \quad x \in \partial B_{0} .
$$

By the maximum principle, one has $\beta(x)-u(x) \geq 0$ for $x \in B_{0}$. This contra$\operatorname{dicts} \beta\left(x_{0}\right)-u\left(x_{0}\right)=-m<0$.

Consequently,

$$
\alpha(x) \leq u(x) \leq \beta(x), \quad x \in \bar{\Omega} .
$$

Step 2. Every solution of (2.3) is a solution of (2.1). Every solution of (2.3) satisfies $\alpha(x) \leq u(x) \leq \beta(x), x \in \bar{\Omega}$. From the definition of $K$ and $\bar{F}$, we have $\bar{F}(x, u(x))=F(x, u(x)), \quad|\nabla u(x)| \leq \frac{1}{a_{0}} \int_{\Omega}\left|G_{x}(x, y)\right| E(y) d y=H(x), \quad x \in \Omega$

and so

$$
a\left(\int_{\Omega}|| \nabla u(x)\left|-(|\nabla u(x)|-H(x))^{+}\right|^{2} d x\right)=a\left(\int_{\Omega}|\nabla u(x)|^{2} d x\right) .
$$

Thus, $u$ is a solution of (2.1).

Step 3. The problem (2.1) has at least one solution.

Since $E \in L^{p}$, there is an $R>0$ such that $\|E\|_{p} \leq R$. From (2.2) and the construction of $\bar{F}$, we have, for every $u \in C^{1}(\bar{\Omega})$,

$$
\left|\frac{\bar{F}(x, u(x))}{a\left(\int_{\Omega}|| \nabla u(x)\left|-(|\nabla u(x)|-H(x))^{+}\right|^{2} d x\right)}\right| \leq \frac{1}{a_{0}} h_{R}(x), \forall x \in \Omega .
$$

Define operators

$$
\bar{N}: C^{1}(\bar{\Omega}) \rightarrow L^{p}(\Omega): u \mapsto \frac{\bar{F}(x, u(x))}{a\left(\int_{\Omega}|| \nabla u(x)\left|-(|\nabla u(x)|-H(x))^{+}\right|^{2} d x\right)}
$$

and $\bar{A}: C^{1}(\bar{\Omega}) \rightarrow C^{1}(\bar{\Omega})$ by

$$
\bar{A} u=(-\Delta)^{-1}(\bar{N} u) .
$$


Note $\bar{A}$ is completely continuous and there exists a $K_{0}>0$ big enough such that for all $v \in \bar{A}\left(C^{1}(\bar{\Omega})\right)$, we have

$$
\|v\| \leq K_{0} .
$$

Then there exists $\bar{K}_{0}>\max \left\{\|\alpha\|,\|\beta\|, K_{0}\right\}$ big enough such that

$$
\bar{A}\left(\overline{B_{C^{1}}\left(0, \bar{K}_{0}\right)}\right) \subseteq B_{C^{1}}\left(0, \bar{K}_{0}\right),
$$

and by a classical result in degree theory

$$
\operatorname{deg}\left(I-\bar{A}, B_{C^{1}}(0, \bar{K}), \theta\right)=1 .
$$

Therefore there exists a $u \in B_{C^{1}}\left(0, \bar{K}_{0}\right)$ such that

$$
u=\bar{A} u .
$$

Now Step 1 and Step 2 yield

$$
\alpha(x) \leq u(x) \leq \beta(x)
$$

and

$$
a\left(\int_{\Omega}|| \nabla u(x)\left|-(|\nabla u(x)|-H(x))^{+}\right|^{2} d x\right)=a\left(\int_{\Omega}|\nabla u(x)|^{2} d x\right), \quad x \in \Omega
$$

and so $u(x)$ is a solution to (2.1).

Step 4. If $\alpha(x)$ and $\beta(x)$ are strict subsolution and supersolution, we show

$$
\operatorname{deg}(I-A, S, \theta)=1 .
$$

Since $\alpha(x)$ and $\beta(x)$ are strict subsolution and supersolution, $A$ has no fixed point on $\partial S$ and so $\operatorname{deg}(I-A, S, \theta)$ is well defined. Since $A$ has no fixed point in $B_{C^{1}}(0, \bar{K})-S$, we have

$$
\operatorname{deg}(I-A, S, \theta)=\operatorname{deg}\left(I-A, B_{C^{1}}(0, \bar{K}), \theta\right)=1 .
$$

The proof is complete.

Now we consider another special problem

$$
\left\{\begin{array}{l}
-a\left(\int_{\Omega}|\nabla u(x)|^{2} d x\right) \Delta u(x)=F(x, u(x))=F_{1}(x, u(x))+F_{2}(x, u(x)), \quad x \text { in } \Omega, \\
u=0, \quad x \text { on } \partial \Omega .
\end{array}\right.
$$


Definition 2.5. The pair functions $\alpha$ and $\beta$ with $\alpha, \beta \in C^{1}(\bar{\Omega}) \cap C^{2}(\Omega)$ are subsolution and supersolution of (2.4) if $\alpha(x) \leq u \leq \beta(x)$ for $x \in \Omega$, and

$$
\begin{aligned}
& \left\{\begin{array}{l}
-\Delta \alpha(x) \leq \frac{1}{b_{0}} F_{1}(x, \alpha(x))+\frac{1}{a_{0}} F_{2}(x, \alpha(x)), \quad x \text { in } \Omega, \\
\left.\alpha\right|_{\partial \Omega} \leq 0,
\end{array}\right. \\
& \left\{\begin{array}{l}
-\Delta \beta(x) \geq \frac{1}{a_{0}} F_{1}(x, \beta(x))+\frac{1}{b_{0}} F_{2}(x, \beta(x)), \\
\left.\beta\right|_{\partial \Omega} \geq 0,
\end{array}\right.
\end{aligned}
$$

where $a_{0}=a(0)$ and $b_{0}=a\left(\int_{\Omega} H(x)^{2} d x\right), E \in L^{p}(\Omega)(p>N)$; here

$$
\begin{gathered}
E(x)=\sup _{u \in[\alpha(x), \beta(x)]}|F(x, u)|, \quad x \in \bar{\Omega}, \\
H(x)=\frac{1}{a_{0}} \int_{\Omega}\left|G_{x}(x, y)\right| E(y) d y, \quad x \in \bar{\Omega}
\end{gathered}
$$

and $G(x, y)$ is the Green's function for $-\Delta u(x)=h$ and $\left.u\right|_{\partial \Omega}=0$.

Theorem 2.2. Let $\Omega \subseteq R^{N}(N \geq 1)$ be a smooth bounded domain. Suppose that $F: \Omega \times R \rightarrow R$ is a continuous function. Assume $\alpha$ and $\beta$ are the subsolution and supersolution of (2.4) respectively. If

$$
F_{1}(x, u) \geq 0, \quad F_{2}(x, u) \leq 0, \quad \forall x \in \Omega, \alpha(x) \leq u \leq \beta(x),
$$

then problem (2.4) has at least one solution $u$ such that, for all $x \in \bar{\Omega}$,

$$
\alpha(x) \leq u(x) \leq \beta(x) .
$$

If moreover $\alpha(x)$ and $\beta(x)$ are strict and satisfy $\alpha \prec \beta$, then

$$
S=\left\{u \in C^{1}(\bar{\Omega}) \mid \alpha \prec \beta\right\} .
$$

is admissible for the degree (for the map $A$ ) and

$$
\operatorname{deg}(I-A, S, \theta)=1 \text {. }
$$

Proof. Let

$$
\begin{aligned}
& \bar{F}_{1}(x, u)=\left\{\begin{array}{l}
F_{1}(x, \alpha(x)), \text { if } u<\alpha(x) ; \\
F_{1}(x, u), \text { if } \alpha(x) \leq u \leq \beta(x) ; \\
F_{1}(x, \beta(x)), \text { if } u>\beta(x)
\end{array}\right. \\
& \bar{F}_{2}(x, u)=\left\{\begin{array}{l}
F_{2}(x, \alpha(x)), \text { if } u<\alpha(x) ; \\
F_{2}(x, u), \text { if } \alpha(x) \leq u \leq \beta(x) ; \\
F_{2}(x, \beta(x)), \text { if } u>\beta(x)
\end{array}\right.
\end{aligned}
$$


and

$$
\bar{F}(x, u)=\bar{F}_{1}(x, u)+\bar{F}_{2}(x, u), \quad \forall(x, u) \in \Omega \times \mathbb{R} .
$$

We will study the modified problem

$$
\left\{\begin{array}{l}
-\Delta u=\frac{\bar{F}(x, u)}{\left.a\left(\int_{\Omega}|| \nabla u(x)\left|-(|\nabla u(x)|-H(x))^{+}\right|\right)^{2} d x\right)}, \quad x \in \Omega \\
\left.u\right|_{\partial \Omega}=0
\end{array}\right.
$$

Step 1. Every solution $u$ of (2.6) satisfies $\alpha(x) \leq u(x) \leq \beta(x), x \in \bar{\Omega}$.

We prove that $\alpha(x) \leq u(x)$ on $\bar{\Omega}$. Obviously, ||$\nabla u(x) \mid-(|\nabla u(x)|-$ $H(x))\left.^{+}\right|^{2} \leq H(x)^{2}$, which together with the monotonicity of $a(t)$ implies that

$$
\left.a_{0} \leq a\left(\int_{\Omega}|| \nabla u(x)\left|-(|\nabla u(x)|-H(x))^{+}\right|\right)^{2} d x\right) \leq a\left(\int_{\Omega} H(x)^{2} d x\right) .
$$

By contradiction, assume that $\max _{x \in \bar{\Omega}}(\alpha(x)-u(x))=M>0$. Note that $\alpha(x)-u(x) \not \equiv M$ on $\bar{\Omega}(\alpha(x)-u(x) \leq 0, x \in \partial \Omega)$. If $x_{0} \in \Omega$ is such that $\alpha\left(x_{0}\right)-u\left(x_{0}\right)=M$, choose $A_{0}=\{x \in \Omega \mid \alpha(x)-u(x)>0\}$ a connected domain with $x_{0} \in A_{0}$. It follows from (2.5) that

$$
\begin{aligned}
& \Delta(\alpha(x)-u(x)) \\
\leq & \frac{1}{b_{0}} F_{1}(x, \alpha(x))+\frac{1}{a_{0}} F_{2}(x, \alpha(x)) \\
& -\frac{1}{a\left(\int_{\Omega}|| \nabla u(x)\left|-(|\nabla u(x)|-H(x))^{+}\right|{ }^{2} d x\right)} \bar{F}(x, u(x)) \\
= & \frac{1}{b_{0}} F_{1}(x, \alpha(x))-\frac{1}{a\left(\int_{\Omega}|| \nabla u(x)\left|-(|\nabla u(x)|-H(x))^{+}\right|^{2} d x\right)} \bar{F}_{1}(x, u(x)) \\
& +\frac{1}{a_{0}} F_{2}(x, \alpha(x))-\frac{1}{a\left(\int_{\Omega}|| \nabla u(x)\left|-(|\nabla u(x)|-H(x))^{+}\right|^{2} d x\right)} \bar{F}_{2}(x, u(x)) \\
\leq & \frac{1}{b_{0}}\left[F_{1}(x, \alpha(x))-F_{1}(x, \alpha(x))\right]+\frac{1}{a_{0}}\left[F_{2}(x, \alpha(x))-F_{2}(x, \alpha(x))\right] \\
= & 0, \quad x \in A_{0}
\end{aligned}
$$

and

$$
\alpha(x)-u(x)=0, \quad x \in \partial A_{0} .
$$

From the maximum principle, one has $\alpha(x)-u(x) \leq 0$ for $x \in A_{0}$. This contradicts $\alpha\left(x_{0}\right)-u\left(x_{0}\right)>0$.

Now we prove that $\beta(x) \geq u(x)$ on $\bar{\Omega}$. By contradiction, assume that $\min _{x \in \bar{\Omega}}(\beta(x)-u(x))=-m<0$. Note that $\beta(x)-u(x) \not \equiv-m$ on $\bar{\Omega}(\beta(x)-$ $u(x) \geq 0, x \in \partial \Omega)$. If $x_{0} \in \Omega$ is such that $\beta\left(x_{0}\right)-u\left(x_{0}\right)=-m$, choose $B_{0}=\{x \in \Omega \mid \beta(x)-u(x)<0\}$ a connected domain with $x_{0} \in B_{0}$. It follows 
from (2.5) that

$$
\begin{aligned}
& -\Delta(\beta(x)-u(x)) \\
\geq & \frac{1}{a_{0}} F_{1}(x, \beta(x))+\frac{1}{b_{0}} F_{2}(x, \beta(x)) \\
& -\frac{1}{a\left(\int_{\Omega}|| \nabla u(x)\left|-(|\nabla u(x)|-H(x))^{+}\right| 2 d x\right)} \bar{F}(x, u(x)) \\
= & \frac{1}{a_{0}} F_{1}(x, \beta(x))-\frac{1}{a\left(\int_{\Omega}|| \nabla u(x)\left|-(|\nabla u(x)|-H(x))^{+}\right|^{2} d x\right)} \bar{F}_{1}(x, u(x)) \\
& +\frac{1}{b_{0}} F_{2}(x, \beta(x))-\frac{1}{a\left(\int_{\Omega}|| \nabla u(x)\left|-(|\nabla u(x)|-H(x))^{+}\right|^{2} d x\right)} \bar{F}_{2}(x, u(x)) \\
\geq & \frac{1}{b_{0}}\left[F_{1}(x, \beta(x))-F_{1}(x, \beta(x))\right]+\frac{1}{a_{0}}\left[F_{2}(x, \beta(x))-F_{2}(x, \beta(x))\right] \\
= & 0, \quad x \in B_{0}
\end{aligned}
$$

and

$$
\beta(x)-u(x)=0, \quad x \in \partial B_{0} .
$$

From the maximum principle, one has $\beta(x)-u(x) \geq 0$ for $x \in B_{0}$. This contradicts $\beta\left(x_{0}\right)-u\left(x_{0}\right)=-m<0$.

Consequently,

$$
\alpha(x) \leq u(x) \leq \beta(x), \quad x \in \bar{\Omega} .
$$

The proof of Step 2-Step 4 are the same as that in the proof of Theorem 2.1 so we omit them.

The proof is complete.

Remark 2.3. The difference between the above two theorems and those in $[6,8,13-14,16,28]$ are:

(1) we remove the monotonicity of $f$ on $u$ in $[6,9,14-15]$;

(2) we define only one subsolution instead of a sequence of subsolutions $\left\{\underline{u}_{\delta}\right\}$ with $\left\|\underline{u}_{\delta}\right\| \rightarrow 0$ as $\delta \rightarrow 0$ as in [14-15];

(3) we obtain the existence of a classical solution instead of a weak solution in $[6,9,14-15,17,31]$;

(4) we give information on how to compute the topological degree.

Remark 2.4. It is also natural to give the following definition of subsupersolutions to $(2.1)$.

Definition 2.1'. The pair functions $\alpha$ and $\beta$ with $\alpha, \beta \in C^{1}(\bar{\Omega}) \cap C^{2}(\Omega)$ are subsolution and supersolution of (2.1) if $\alpha(x) \leq u(x) \leq \beta(x)$ for $x \in \Omega$, and

$$
\left\{\begin{array}{l}
-\Delta \alpha(x) \leq \frac{1}{a\left(\int_{\Omega}|\nabla \alpha(x)|^{2} d x\right)} F(x, \alpha(x)), \quad x \text { in } \Omega, \\
\left.\alpha\right|_{\partial \Omega} \leq 0,
\end{array}\right.
$$




$$
\left\{\begin{array}{l}
-\Delta \beta(x) \geq \frac{1}{a\left(\int_{\Omega}|\nabla \beta(x)|^{2} d x\right)} F(x, \beta(x)), \quad x \text { in } \Omega, \\
\left.\beta\right|_{\partial \Omega} \geq 0 .
\end{array}\right.
$$

We give an example which illustrates that perhaps there is no solution between the subsolution and supersolution if we use Definition 2.1'.

Example 2.1. We consider the following nonlocal problem

$$
\left\{\begin{array}{l}
-u^{\prime \prime}(t)=\frac{1}{a\left(\int_{\Omega}\left|u^{\prime}(t)\right|^{2} d t\right)} \cdot 1, \quad t \in(0,1), \\
u(0)=u(1)=0
\end{array}\right.
$$

where

$$
a(t)=t, \quad t \in[0,+\infty) .
$$

Obviously, the following problem

$$
\left\{\begin{array}{l}
-u^{\prime \prime}(t)=1, \quad t \in(0,1) \\
u(0)=u(1)=0
\end{array}\right.
$$

has a unique positive solution $e(t)=\frac{1}{2} t(1-t), t \in[0,1]$.

Now we show (2.7) has a unique positive solution.

Let

$$
G(s)=s a\left(\frac{s^{2}}{12}\right)-1, \quad t \in[0,+\infty) .
$$

It easy to see that $G(s)$ is increasing on $[0,+\infty)$ with

$$
G(0)=-1, \quad \lim _{s \rightarrow+\infty} G(s)=+\infty
$$

which guarantees that there exists a unique $s_{0}>0$ such that $G\left(s_{0}\right)=0$, i.e.,

$$
s_{0}=\frac{1}{a\left(\frac{s_{0}^{2}}{12}\right)} \text {. }
$$

Let $u(t)=s_{0} e(t), t \in(0,1)$. Then

$$
\left\{\begin{aligned}
-u^{\prime \prime}(t) & =-\left(s_{0} e(t)\right)^{\prime \prime} \\
& =s_{0} \\
& =\frac{1}{a\left(\frac{s_{0}^{2}}{12}\right)} \\
& =\frac{1}{a\left(\int_{0}^{1}\left|u^{\prime}(t)\right|^{2} d t\right)} \cdot 1, \quad t \in(0,1), \\
u(0) & =u(1)=0,
\end{aligned}\right.
$$

i.e., (2.7) has at least one positive solution $u(t)=s_{0} e(t)$. 
Now let $u_{0}(t)$ be a positive solution of $(2.7)$. Let $\lambda_{0}=\frac{1}{a\left(\int_{0}^{1}\left|u_{0}^{\prime}(t)\right|^{2} d t\right)}$. Then

$$
\left\{\begin{array}{l}
-u_{0}^{\prime \prime}(t)=\lambda_{0}, \quad t \in(0,1) \\
u_{0}(0)=u_{0}(1)=0
\end{array}\right.
$$

which implies $u_{0}(t)=\lambda_{0} e(t), t \in[0,1]$ and

$$
\lambda_{0}=\frac{1}{a\left(\int_{0}^{1}\left|u_{0}^{\prime}(t)\right|^{2} d t\right)}=\frac{1}{a\left(\int_{0}^{1}\left|\lambda_{0} e^{\prime}(t)\right|^{2} d t\right)}=\frac{1}{a\left(\lambda_{0}^{2} \frac{1}{12}\right)}
$$

Since $G(s)=0$ has a unique positive solution $s_{0}$, one has $\lambda_{0}=s_{0}$.

Consequently, (2.7) has a unique positive solution.

Next we construct sub-supersolutions which satisfy Definition 2.1'. Let $\bar{u}(t)=2 s_{0} e(t)$. The monotonicity of $G$ guarantees that

$$
2 s_{0}>\frac{1}{a\left(\frac{\left(2 s_{0}\right)^{2}}{12}\right)}=\frac{1}{a\left(\int_{0}^{1}\left|\bar{u}^{\prime}(t)\right|^{2} d t\right)} \text {. }
$$

Then

$$
\left\{\begin{aligned}
-\bar{u}^{\prime \prime}(t) & =-\left(2 s_{0} e(t)\right)^{\prime \prime} \\
& =2 s_{0} \\
& >\frac{1}{a\left(\int_{0}^{1}\left|\bar{u}_{0}^{\prime}(t)\right|^{2} d t\right)} \cdot 1, \quad t \in(0,1), \\
\bar{u}(0) & =\bar{u}(1)=0,
\end{aligned}\right.
$$

i.e., $\bar{u}$ is supersolution to (2.7) satisfying Definition $2.1^{\prime}$.

For $0<\varepsilon<\frac{1}{4}$, let

$$
\begin{gathered}
a=-\frac{s_{0}}{3 \varepsilon}, \quad b=2 s_{0}, \\
c=2 s_{0} e^{\prime}(\varepsilon)-3 a \varepsilon^{2}-2 b \varepsilon=s_{0}(1-2 \varepsilon)-3 a \varepsilon^{2}-2 b \varepsilon
\end{gathered}
$$

and

$$
d=2 s_{0} e(\varepsilon)-\left(a \varepsilon^{3}+b \varepsilon^{2}+c \varepsilon\right)
$$

Let

$$
f(t)=a t^{3}+b t^{2}+c t+d, \quad t \in[\varepsilon, 2 \varepsilon] .
$$

It is easy to see that from

$$
f^{\prime \prime \prime}(t)=6 a<0, \quad t \in[\varepsilon, 2 \varepsilon]
$$

and

$$
f^{\prime \prime}(\varepsilon)=2 s_{0}, \quad f^{\prime \prime}(2 \varepsilon)=0,
$$

one has

$$
0<f^{\prime \prime}(t)<2 s_{0}, \quad\left|f^{\prime}(t)\right| \leq\left|f^{\prime}(\varepsilon)\right|+2 s_{0} \varepsilon, \quad t \in[\varepsilon, 2 \varepsilon]
$$


Let

$$
f_{1}(t)=f(1-t), \quad t \in[1-2 \varepsilon, 1-\varepsilon] .
$$

Clearly

$$
0<f_{1}^{\prime \prime}(t)=f^{\prime \prime}(1-t)<2 s_{0}, \quad\left|f^{\prime}(t)\right| \leq\left|f^{\prime}(\varepsilon)\right|+2 s_{0} \varepsilon, \quad t \in[1-2 \varepsilon, 1-\varepsilon] .
$$

For $0<\varepsilon<\frac{1}{4}$, let

$$
\underline{u}_{\varepsilon}(t)=\left\{\begin{array}{l}
2 s_{0} e(t), \quad t \in[0, \varepsilon] \\
f(t), \quad t \in[\varepsilon, 2 \varepsilon] \\
f(\varepsilon), \quad t \in[2 \varepsilon, 1-2 \varepsilon] \\
f_{1}(t), \quad t \in[1-2 \varepsilon, 1-\varepsilon] \\
2 s_{0} e(t), \quad t \in[1-\varepsilon, 1]
\end{array}\right.
$$

Now (2.8) and (2.9) guarantee that

$$
\left\{\begin{array}{l}
-u_{\varepsilon}^{\prime \prime}(t) \leq 2 s_{0}, \\
\int_{0}^{1}\left|u_{\varepsilon}^{\prime}(t)\right|^{2} d t=\int_{0}^{2 \varepsilon}\left|u_{\varepsilon}^{\prime}(t)\right|^{2} d t+\int_{1-2 \varepsilon}^{1}\left|u_{\varepsilon}^{\prime}(t)\right|^{2} d t \rightarrow 0, \quad \text { as } \varepsilon \rightarrow 0 .
\end{array}\right.
$$

Choose $\varepsilon_{0}>0$ small enough such that

$$
2 s_{0} a\left(\int_{0}^{1}\left|u_{\varepsilon_{0}}^{\prime}(t)\right|^{2} d t\right)<1,
$$

which together with (2.10) implies that

$$
\left\{\begin{aligned}
-\bar{u}_{\varepsilon_{0}}^{\prime \prime}(t) & \leq 2 s_{0} \\
& <\frac{1}{a\left(\int_{0}^{1}\left|\underline{u}_{\varepsilon_{0}}^{\prime}(t)\right|^{2} d t\right)} \cdot 1, \quad t \in(0,1), \\
\underline{u}_{\varepsilon_{0}}(0) & =\underline{u}_{\varepsilon_{0}}(1)=0
\end{aligned}\right.
$$

i.e., $\underline{u}_{\varepsilon_{0}}$ is sub-solution to (2.7) satisfying Definition 2.1'.

Finally, we show there is no solution between $\bar{u}$ and $\underline{u}_{\varepsilon_{0}}$.

In fact, suppose that $u_{0}$ is a positive solution to $(2.7)$ between $\bar{u}$ and $\underline{u}_{\varepsilon_{0}}$. It is easy to see that

$$
u_{0}(t)=2 s_{0} e(t) \neq s_{0} e(t), \quad t \in[0, \varepsilon],
$$

which implies that $u_{0} \neq s_{0} e$. However we know that (2.7) has a unique positive solution $s_{0} e(t)$. This is a contradiction. 


\section{The positive solutions when $f(x, u)=K(x) u^{-p}$}

In this section, we consider the singular problems

$$
\left\{\begin{array}{l}
-a\left(\int_{\Omega}|\nabla u(x)|^{2} d x\right) \Delta u(x)=K(x) u(x)^{-p}, \quad x \text { in } \Omega, \\
u>0, \quad x \text { in } \Omega \\
u=0, \quad x \text { on } \partial \Omega
\end{array}\right.
$$

where $\Omega \subseteq R^{N}$ is a smooth bounded domain. Let $d(x)=\min \{d(x, \partial \Omega) \mid x \in \bar{\Omega}\}$.

Let $e \in C^{2, \alpha}(\bar{\Omega})$ be defined by

$$
-\Delta u=1, \quad x \in \Omega ; u(x)=0, x \in \partial \Omega
$$

with $0 \leq e(x) \leq 1$ for all $x \in \bar{\Omega}$ and let $\Phi_{1}$ is the eigenfunction with $0 \leq$ $\Phi_{1}(x) \leq 1$ for $x \in \bar{\Omega}$ corresponding to the principle eigenvalue $\lambda_{1}$ of

$$
\left\{\begin{array}{l}
-\Delta u=\lambda u, \quad x \in \Omega, \\
\left.u\right|_{\partial \Omega}=0
\end{array}\right.
$$

Note that $\lambda_{1}>0, \Phi_{1}(x)>0$ for $x \in \Omega$ and

$$
\left|\nabla \Phi_{1}(x)\right|>0, \quad \forall x \in \partial \Omega
$$

From [39], the following results is true

$$
\frac{\Phi_{1}}{e} \in C(\bar{\Omega}) .
$$

Now we note the following conditions:

$\left(\mathrm{H}_{1}\right) K \in C(\bar{\Omega}, R)$ with $K(x)>0$ for all $x \in \Omega$ and

$$
\text { there exists a } 1>\tau \geq 0 \text { such that } K[d(x)]^{\tau-p} \in L^{\infty}(\Omega) \text {, }
$$

$\left(\mathrm{H}_{2}\right)$

$$
\lim _{t \rightarrow+\infty} \frac{t}{a(t)^{2(p-1)}}=+\infty .
$$

Theorem 3.1. If $\left(H_{1}\right)-\left(H_{2}\right)$ hold, (3.1) has a unique positive solution $u \in C^{2+\alpha}(\Omega) \cap C(\bar{\Omega})$ with $u(x)>0$ for all $x \in \Omega$. If $p>1$, then there exist positive constants $b_{1}$ and $b_{2}$ such that $b_{1} \Phi_{1}(x)^{\frac{2}{1+p}} \leq u(x) \leq b_{2} \Phi_{1}(x)^{\frac{2}{1+p}}$.

Proof. The proof is based on Theorem 2.1 and we construct the pairs of sub-supersolutions. The construction of supersolutions to (1.1) when $p>1$ is different from that when $0<p \leq 1$. 
(1) Assume first that $p>1$. In this case, let $t=2 /(1+p)$ and let $\Psi(x)=$ $b \Phi_{1}(x)^{t}$ where $b>0$ is a constant. From (3.3), we deduce that

$$
\Delta \Psi(x)+q(x, b) \Psi^{-p}(x)=0, \quad x \in \Omega,
$$

where $q(x, b)=b^{1+p}\left[t(1-t)\left|\nabla \Phi_{1}(x)\right|^{2}+t \lambda_{1} \Phi_{1}(x)^{2}\right]$. Since $0<t<1$, from (3.4), choose a positive constant $b$ such that

$$
\frac{1}{a_{0}} K(x)<q(x, b), \quad \forall x \in \Omega
$$

Let $u(x)=b \Phi_{1}(x)^{t}$. Hence,

$$
\Delta u(x)+\frac{1}{a_{0}} K(x) u(x)^{-p}=\left[\frac{1}{a_{0}} K(x)-q(x, b)\right] u^{-p}(x)<0, \quad x \in \Omega .
$$

(2) Assume that $0<p \leq 1$. Let $s$ be chosen to satisfy the two inequalities

$$
0<s<1, s(1+p)<2
$$

Let $u(x)=c \Phi_{1}(x)^{s}$, where $c$ is a large positive constant to be chosen below. For $x \in \Omega$, we have

$$
\begin{aligned}
& \Delta u(x)+\frac{1}{a_{0}} K(x) u(x)^{-p} \\
& =-\Phi_{1}(x)^{s-2}\left[\left|\nabla \Phi_{1}(x)\right|^{2} c s(1-s)-\frac{1}{a_{0}} K(x) c^{-p} \Phi_{1}(x)^{2-(1+p) s}\right]-c \lambda_{1} s \Phi_{1}(x)^{-p} .
\end{aligned}
$$

Since the inequalities (3.10) hold, we can choose $c>0$ so large that

$$
\begin{aligned}
\Delta u(x)+\frac{1}{a_{0}} K(x) u(x)^{p}= & -\Phi_{1}(x)^{s-2}\left[\left|\nabla \Phi_{1}(x)\right|^{2} c s(1-s)\right. \\
& \left.-\frac{1}{a_{0}} K(x) c^{-p} \Phi_{1}(x)^{2-(1+p) s}\right]-c \lambda_{1} s \Phi_{1}(x)^{-p} \\
< & 0, \quad x \in \Omega .
\end{aligned}
$$

Choose $d=\max \{b, c\}$ and define

$$
u^{*}(x)= \begin{cases}d \Phi_{1}^{t}(x), & x \in \bar{\Omega} \text { if } p>1 \\ d \Phi_{1}^{s}(x), & x \in \bar{\Omega} \text { if } 0<p \leq 1\end{cases}
$$

From (3.11) and (3.9), we have

$$
\Delta u^{*}(x)+\frac{1}{a_{0}} K(x) u^{*}(x)^{-p}<0, \quad \forall x \in \Omega .
$$


It follows that for each $n \in \mathbb{N}$

$\Delta u^{*}(x)+\frac{1}{a_{0}} K(x)\left(u^{*}(x)+\frac{1}{n}\right)^{-p}<\Delta u^{*}(x)+\frac{1}{a_{0}} K(x) u^{*}(x)^{-p}<0, \quad \forall x \in \Omega$.

Let $u_{*}(x)=0, x \in \bar{\Omega}$ and let

$$
E_{n}(x)=K(x)\left(\frac{1}{n}\right)^{-p}, \quad H_{n}(x)=\frac{1}{a_{0}} \int_{\Omega}\left|G_{x}(x, y)\right| E_{n}(y) d y, \quad x \in \bar{\Omega}
$$

and

$$
b_{n}=a\left(\int_{\Omega} H_{n}^{2}(x) d x\right) .
$$

From the definitions of $u_{*}$ and $u^{*}$, for $n \in \mathbb{N}=\{1,2, \cdots\}$, from (3.12), we have for each $n \in \mathbb{N}$

$$
\left\{\begin{array}{l}
\Delta u^{*}(x)+\frac{1}{a_{0}}\left(u^{*}(x)+\frac{1}{n}\right)^{-p}<0, \quad x \in \Omega, \\
\left.u^{*}\right|_{\partial \Omega}=0
\end{array}\right.
$$

and

$$
\left\{\begin{array}{l}
\Delta u_{*}(x)+\frac{1}{b_{n}}\left(u_{*}(x)+\frac{1}{n}\right)^{-p}>0, \quad x \in \Omega, \\
\left.u^{*}\right|_{\partial \Omega}=0 .
\end{array}\right.
$$

Now Theorem 2.1 guarantees that for $n \in \mathbb{N}$, there exist $\left\{u_{n}\right\}$ with $u_{*}(x) \leq$ $u_{n}(x) \leq u^{*}(x)$ for all $x \in \bar{\Omega}$ such that

$$
\left\{\begin{array}{l}
\Delta u_{n}(x)+\frac{1}{a\left(\int_{\Omega}\left|\nabla u_{n}(x)\right|^{2} d x\right)} K(x)\left(u_{n}(x)+\frac{1}{n}\right)^{-p}=0, \quad x \in \Omega, \\
\left.u_{n}\right|_{\partial \Omega}=0 .
\end{array}\right.
$$

Choose an $L>0$ such that

$$
0 \leq u_{n}(x)+\frac{1}{n} \leq L, \quad \forall x \in \bar{\Omega} .
$$

It follows that

$$
\begin{aligned}
u_{n}(x) & =\frac{1}{a\left(\left\|u_{n}\right\|^{2}\right)} \int_{\Omega} G(x, y) K(y)\left(u_{n}(y)+\frac{1}{n}\right)^{-p} d y \\
& \geq \frac{1}{a\left(\left\|u_{n}\right\|^{2}\right)} \int_{\Omega} G(x, y) K(y) d y L^{-p} .
\end{aligned}
$$

Now we show that $\left\{\left\|u_{n}\right\|\right\}$ is bounded. 
From the definition of $\Phi_{1}$, Theorem 2.2 in [16] implies that there exists a $\theta_{1}>0$ and $\theta_{2}>0$ such that

$$
\theta_{1} d(x) \leq \int_{\Omega} G(x, z) K(z) d z \leq \theta_{2} d(x), \quad x \in \bar{\Omega},
$$

which together with (3.14) yields that

$$
\begin{aligned}
\frac{\partial u}{\partial x_{i}}(x) & =\frac{1}{a\left(\left\|u_{n}\right\|^{2}\right)} \int_{\Omega} G_{x_{i}}(x, y) K(y)\left(u_{n}(y)+\frac{1}{n}\right)^{-p} d y \\
& \leq \frac{1}{a\left(\left\|u_{n}\right\|^{2}\right)} \int_{\Omega}\left|G_{x_{i}}(x, y)\right| K(y)\left(\frac{1}{a\left(\left\|u_{n}\right\|^{2}\right)} \int_{\Omega} G(y, z) K(z) d z L^{-p}\right)^{-p} d y \\
& =L^{p^{2}} a\left(\left\|u_{n}\right\|^{2}\right)^{p-1} \int_{\Omega}\left|G_{x_{i}}(x, y)\right| K(y) \theta_{1}^{-p} d(y)^{-p} d y, \quad i=1,2, \cdots, N
\end{aligned}
$$

Then

$$
\left.\|u\|^{2} \leq L^{2 p^{2}} a\left(\left\|u_{n}\right\|^{2}\right)^{2(p-1)} \int_{\Omega} \sum_{i=1}^{2}\left(\int_{\Omega} G_{x_{i}}(x, y) K(y) \theta_{1}^{-p} d(y)^{-p} d y\right)^{2}\right) d x
$$

i.e.,

$$
\left.\frac{\|u\|^{2}}{a\left(\|u\|^{2}\right)^{2(p-1)}} \leq L^{2 p^{2}} \int_{\Omega} \sum_{i=1}^{2}\left(\int_{\Omega} G_{x_{i}}(x, y) K(y) \theta_{1}^{-p} d(y)^{-p} d y\right)^{2}\right) d x
$$

which together with (3.7) implies that there exists a $\alpha_{0}>0$ such

$$
\left\|u_{n}\right\| \leq \alpha_{0}, \quad n=1,2, \cdots
$$

From (3.14) and the monotonicity of $a(t)$, one has

$$
u_{n}(x) \geq \frac{1}{a\left(\alpha_{0}\right)} \int_{\Omega} G(x, y) K(y) d y L^{-p} \stackrel{\text { def. }}{=} v_{0}(x), \quad n=1,2, \cdots
$$

Let $\Omega_{k}=\left\{x \in \Omega \mid v_{0}(x)>\frac{1}{k}\right\}, k \in \mathbb{N}$. From (3.13), we have

$$
\left|\Delta u_{n}(x)\right| \leq \frac{1}{a_{0}} K(x) v_{0}(x)^{-p} \leq \frac{1}{a_{0}} \max _{x \in \bar{\Omega}} K(x)\left(\min _{x \in \bar{\Omega}_{k}} v_{0}(x)\right)^{-p}, \quad x \in \bar{\Omega}_{k},
$$

which implies that

$\left\{u_{n}(x)\right\}$ is equicontinous and uniformly bounded on $\bar{\Omega}_{k}, \quad k \in \mathbb{N}$.

and

$\left\{\nabla u_{n}(x)\right\}$ is equicontinous and uniformly bounded on $\bar{\Omega}_{k}, \quad k \in \mathbb{N}$. 
Therefore, $\left\{u_{n}(x)\right\}$ has a uniformly convergent subsequence $\left\{u_{n}^{(k)}(x)\right\}$ on every $\bar{\Omega}_{k}$ and $\left\{\nabla u_{n}^{(k)}(x)\right\}$ converges uniformly on $\bar{\Omega}_{k}$ also. From the diagonal method, we can choose a subsequence $\left\{u_{n, k}^{(k)}(x)\right\}$ of $\left\{u_{n}(x)\right\}$ which converges to a $u_{0}$ on every $\bar{\Omega}_{k}$ uniformly and $\left\{\nabla u_{n, k}^{(k)}(x)\right\}$ converges uniformly on $\bar{\Omega}_{k}$ also. Without loss of generality, assume that

$$
\lim _{n \rightarrow+\infty} u_{n}(x)=u_{0}(x), \quad \text { uniformly on } \bar{\Omega}_{k}, \quad k \in \mathbb{N}
$$

and

$$
\lim _{n \rightarrow+\infty} \nabla u_{n}(x)=\nabla u_{0}(x), \quad \text { uniformly on } \bar{\Omega}_{k}, \quad k \in \mathbb{N} .
$$

Obviously,

$$
v_{0}(x) \leq u_{0}(x) \leq u^{*}(x), \quad \forall x \in \Omega
$$

which implies

$$
u_{0}(x)=0, \quad x \in \partial \Omega \text {. }
$$

Moreover, from

$$
\left|\nabla u_{n}(x)\right| \leq \frac{1}{a_{0}} \int_{\Omega}\left|G_{x}(x, y)\right| K(y)\left[v_{0}(y)^{-p}\right] d y, \quad x \in \Omega,
$$

the Dominated Convergence Theorem implies that

$$
\lim _{n \rightarrow+\infty} \int_{\Omega}\left|\nabla u_{n}(x)\right|^{2} d x=\int_{\Omega}\left|\nabla u_{0}(x)\right|^{2} d x,
$$

which together with the continuity of $a(t)$ yields

$$
\lim _{n \rightarrow+\infty} a\left(\int_{\Omega}\left|\nabla u_{n}(x)\right|^{2} d x\right)=a\left(\int_{\Omega}\left|\nabla u_{0}(x)\right|^{2} d x\right) .
$$

Letting $n \rightarrow+\infty$ in (3.13), we have

$$
\left\{\begin{array}{l}
\Delta u_{0}(x)+\frac{1}{a\left(\int_{\Omega}\left|\nabla u_{0}(x)\right|^{2} d x\right)} K(x) u_{0}(x)^{-p}>0, \quad x \in \Omega, \\
\left.u_{0}\right|_{\partial \Omega}=0 .
\end{array}\right.
$$

From Theorem 1 in [22], if $p>1$, there exist a $b_{1}>0$ and $b_{2}>0$ such that

$$
b_{1} \Phi_{1}(x)^{\frac{2}{1+p}} \leq u_{0} \leq b_{2} \Phi_{1}(x)^{\frac{2}{1+p}}, \quad \forall x \in \bar{\Omega} .
$$

We consider the uniqueness of positive solution of (3.1). Assume that $u_{1}$ and $u_{2}$ are two positive solutions. Let $c_{i}=\left(a\left(\int_{\Omega}\left|\nabla u_{i}(x)\right|^{2} d x\right)\right)^{1 /(p+1)}$ and $v_{i}=c_{i} u_{i}, i=1,2$. Then $v_{i}$ satisfies

$$
\left\{\begin{array}{l}
-\Delta v_{i}=K(x) v_{i}^{-p} \\
\left.v_{i}\right|_{\partial \Omega}=0
\end{array}\right.
$$


It is easy to see that Theorem 3.4 in [16] guarantees that

$$
\left\{\begin{array}{l}
-\Delta v=K(x) v^{-p} \\
\left.v\right|_{\partial \Omega}=0
\end{array}\right.
$$

has a unique positive solution, which implies $v_{1}=v_{2}$, i.e.

$$
\begin{aligned}
& \left(a\left(\int_{\Omega}\left|\nabla u_{1}(x)\right|^{2} d x\right)\right)^{1 /(p+1)} u_{1}(x) \\
& =\left(a\left(\int_{\Omega}\left|\nabla u_{2}(x)\right|^{2} d x\right)\right)^{1 /(p+1)} u_{2}(x), \forall x \in \bar{\Omega},
\end{aligned}
$$

and so

$$
\begin{aligned}
& \left(a\left(\int_{\Omega}\left|\nabla u_{1}(x)\right|^{2} d x\right)\right)^{1 /(p+1)} \frac{\partial u_{1}(x)}{\partial x_{i}} \\
& =\left(a\left(\int_{\Omega}\left|\nabla u_{2}(x)\right|^{2} d x\right)\right)^{1 /(p+1)} \frac{\partial u_{2}(x)}{\partial x_{i}}, \forall x \in \bar{\Omega}, \quad i=1,2, \cdots, N .
\end{aligned}
$$

Hence,

$$
\begin{aligned}
& \left(a\left(\int_{\Omega}\left|\nabla u_{1}(x)\right|^{2} d x\right)\right)^{2 /(p+1)}\left|\nabla u_{1}(x)\right|^{2} \\
& =\left(a\left(\int_{\Omega}\left|\nabla u_{2}(x)\right|^{2} d x\right)\right)^{2 /(p+1)}\left|\nabla u_{2}(x)\right|^{2}, \quad \forall x \in \Omega .
\end{aligned}
$$

Integration in $\Omega$ yields that

$$
\begin{aligned}
& \left(a\left(\int_{\Omega}\left|\nabla u_{1}(x)\right|^{2} d x\right)\right)^{2 /(p+1)} \int_{\Omega}\left|\nabla u_{1}(x)\right|^{2} d x \\
& =\left(a\left(\int_{\Omega}\left|\nabla u_{2}(x)\right|^{2} d x\right)\right)^{2 /(p+1)} \int_{\Omega}\left|\nabla u_{2}(x)\right|^{2} d x .
\end{aligned}
$$

The monotonicity of $a$ implies that $(a(t))^{2 /(p+1)} t$ is increasing on $[0,+\infty)$, which guarantees that

$$
\int_{\Omega}\left|\nabla u_{1}(x)\right|^{2} d x=\int_{\Omega}\left|\nabla u_{2}(x)\right|^{2} d x
$$

and so

$$
\left(a\left(\int_{\Omega}\left|\nabla u_{1}(x)\right|^{2} d x\right)\right)^{1 /(p+1)}=\left(a\left(\int_{\Omega}\left|\nabla u_{2}(x)\right|^{2} d x\right)\right)^{1 /(p+1)}
$$


which together with (3.15) yields that $u_{1}(x)=u_{2}(x)$. The proof is complete.

In fact, using an idea in [9], we get a result even if $a(t)$ is not increasing. Assume that $v$ is a positive solution to the following problem

$$
\left\{\begin{array}{l}
\Delta u(x)+K(x) u(x)^{-p}=0, \quad x \in \Omega, \\
\left.u\right|_{\partial \Omega}=0
\end{array}\right.
$$

and $c_{0}=\int_{\Omega}|\nabla v(x)|^{2} d x$.

Theorem 3.2. Suppose that $K \in C(\bar{\Omega})$ with $K(x)>0$ for all $x \in \Omega$. Then (3.1) has at least one positive solution $u \in C^{2+\alpha}(\Omega) \cap C(\bar{\Omega})$ if $a(t)$ is continuous on $[0,+\infty)$ with $a(t) \geq a_{0}=a(0)$. Moreover, the number of positive solutions of (3.1) is the number of positive solutions of the following algebraic equation

$$
t^{p+1} a\left(t^{2} c_{0}\right)=1 .
$$

Proof. From [16] and [22], problem (3.16) has a unique positive solution $v$. If $u$ is a positive solution to (3.1), we define $\lambda=a\left(\int_{\Omega}|\nabla u|^{2} d x\right)$ and $v_{0}=$ $\lambda^{1 /(p+1)} u$. Then we have

$$
\left\{\begin{aligned}
-\Delta v_{0}(x) & =-\lambda^{1 /(p+1)} \Delta u(x) \\
& =\lambda^{1 /(p+1)} \frac{1}{\lambda} K(x) u^{-p}(x) \\
& =\lambda^{1 /(p+1)} \frac{1}{\lambda} K(x) \lambda^{p /(p+1)} v_{0}^{-p}(x) \\
& =K(x) v_{0}^{-p}(x), \quad x \in \Omega, \\
\left.v_{0}\right|_{\partial \Omega}=0, &
\end{aligned}\right.
$$

i.e., $v_{0}(x) \equiv v(x), x \in \bar{\Omega}$. This shows that

each positive solution $u(x)$ of (3.1) can be denoted by $t v(x), x \in \bar{\Omega}$. (3.17)

Since $a(t) \geq a_{0}>0$, we have

$$
\lim _{t \rightarrow 0+} t^{p+1} a\left(t^{2} c_{0}\right)=0, \quad \lim _{t \rightarrow+\infty} t^{p+1} a\left(t^{2} c_{0}\right)=+\infty,
$$

which implies that there exists a $t_{0}>0$ such that $t_{0}^{p+1} a\left(t_{0}^{2} c_{0}\right)=1$. Let 
$u(x)=t_{0} v(x)$. Then

$$
\left\{\begin{aligned}
-\Delta u(x) & =-t_{0} \Delta v(x) \\
& =t_{0} K(x) v^{-p}(x) \\
& =t_{0} t_{0}^{p} K(x) u^{-p}(x) \\
& =\frac{1}{a\left(t_{0}^{2} c_{0}\right)} K(x) u^{-p}(x) \\
& =\frac{1}{a\left(\int_{\Omega}|\nabla u(x)|^{2} d x\right)} K(x) u^{-p}(x), \quad x \in \Omega, \\
\left.u\right|_{\partial \Omega}=0, &
\end{aligned}\right.
$$

i.e, $u(x)$ is a positive solution to (3.1). Moreover, (3.17) guarantees that the number of positive solutions of (3.1) is the number of positive solutions of the following algebraic equation

$$
t^{p+1} a\left(t^{2} c_{0}\right)=1
$$

The proof is complete.

We give an example which illustrates that the term $a(t)$ can leads to the existence of an infinite number of positive solutions to (3.1).

Assume that $p>1$ and $\int_{\Omega}\left|\nabla \Phi_{1}^{t}(x)\right|^{2} d x=c_{0}^{\prime}>0$ and

$$
a(t)=\left\{\begin{array}{lr}
3, & t=0 \\
2+\left(t^{-\frac{1+p}{2}} c_{0}^{\prime \frac{p+1}{2}}-2\right)\left[\sin \left(t^{\frac{1}{2}} c_{0}^{\prime-\frac{1}{2}}\right)\right]^{1+p}, & t>0 .
\end{array}\right.
$$

Obviously, $a(t)$ is not monotone on $[0,+\infty)$. For $b_{k}=2 k \pi+\frac{\pi}{2}$, we have

$$
a\left(b_{k}^{2} c_{0}^{\prime}\right)=2+\left(\left(b_{k}^{2} c_{0}^{\prime}\right)^{-\frac{1+p}{2}} c_{0}^{\prime \frac{p+1}{2}}-2\right)\left[\sin \left(\left(b_{k}^{2} c_{0}^{\prime}\right)^{\frac{1}{2}} c_{0}^{\prime-\frac{1}{2}}\right)\right]^{1+p}=b_{k}^{-(1+p)}, \quad k \in \mathbb{N} .
$$

Let $u_{k}(x)=b_{k} \Phi_{1}(x)^{t}$ and $K(x)=\left[t(1-t)\left|\nabla \Phi_{1}(x)\right|^{2}+t \lambda_{1} \Phi_{1}(x)^{2}\right], x \in \bar{\Omega}$. Clearly, we have

$$
\left\{\begin{array}{l}
\Delta u_{k}(x)+\frac{1}{a\left(\int_{\Omega}\left|\nabla u_{k}(x)\right|^{2} d x\right)} K(x) u_{k}(x)^{-p}=0, \quad x \in \Omega, \\
\left.u_{k}\right|_{\partial \Omega}=0,
\end{array}\right.
$$

i.e.,

$$
\left\{\begin{array}{l}
\Delta u(x)+\frac{1}{a\left(\int_{\Omega}|\nabla u(x)|^{2} d x\right)} K(x) u(x)^{-p}=0, \quad x \in \Omega, \\
\left.u\right|_{\partial \Omega}=0,
\end{array}\right.
$$

has an infinite number of positive solutions. 


\section{The positive solutions when $f(x, u)$ is sign-changing in} $u$

In this section, we consider the following problems

$$
\left\{\begin{array}{l}
-a\left(\int_{\Omega}|\nabla u(x)|^{2} d x\right) \Delta u(x)=\lambda u^{q}(x)-u^{p+1}, \quad x \text { in } \Omega \\
u>0, \quad x \text { in } \Omega \\
u=0, \quad x \text { on } \partial \Omega
\end{array}\right.
$$

where $\Omega \subseteq R^{N}$ is a smooth bounded domain, $q \in(0,1)$ and $p>0$ and

$$
\left\{\begin{array}{l}
-a\left(\int_{\Omega}|\nabla u(x)|^{2} d x\right) \Delta u(x)=\lambda u+f(u)-u^{p+1}, \quad x \text { in } \Omega \\
u>0, \quad x \text { in } \Omega \\
u=0, \quad x \text { on } \partial \Omega .
\end{array}\right.
$$

where $\Omega \subseteq R^{N}$ is a smooth bounded domain, $\lambda \geq 0, p>0, f(u)$ is a nonnegative function of $C^{1}$ class for $u \geq 0$ such that $f(0)=0, f^{\prime}(0)=0$ and $\lim _{u \rightarrow+\infty} f(u) / u^{p+1}=0$. Define

$$
g(u)=\frac{f(u)}{u}-u^{p}, \quad u \geq 0 .
$$

It is easy to see that $g(0)=0$ and $\lim _{u \rightarrow+\infty} g(u) / u^{p}=-1$ and then $\lim _{u \rightarrow+\infty} g(u)=$ $-\infty$. Let

$$
g_{\infty}=\sup _{u \geq 0} g(u)
$$

Theorem 4.1. If $q \in(0,1)$ and $p>0,(4.1)_{\lambda}$ has at least one positive solutions in $C^{2}(\Omega) \cap C^{1}(\bar{\Omega})$ for $\lambda>0$.

Proof. For given $\lambda \in(0,1]$, since $q \in(0,1)$, we can choose $k_{2}>0$ big enough such that

$$
k_{2}>\frac{1}{a_{0}} k_{2}^{q} .
$$

Let $\beta(x)=k_{2} e(x), \quad x \in \bar{\Omega}$, where $e$ is defined in (3.2). Define

$$
H_{1}(x)=\frac{1}{a_{0}} \int_{\Omega}\left|G_{x}(x, y)\right| d y\left(k_{2}^{q}+k_{2}^{p+1}\right), \quad x \in \bar{\Omega}
$$

and

$$
b_{1}=a\left(\int_{\Omega} H_{1}^{2}(x) d x\right) .
$$


Since $q \in(0,1)$ and $p \in(0,+\infty)$, by $(3.5)$, we can choose $\varepsilon>0$ small enough such that

$$
\lambda_{1}<\frac{\lambda}{b_{1}} \varepsilon^{q-1} \Phi_{1}^{q-1}(x)-\frac{1}{a_{0}} \varepsilon^{p} \Phi_{1}^{p}(x), \quad \forall x \in \Omega
$$

and

$$
\varepsilon \Phi_{1}(x)<k_{2} e(x), \quad \forall x \in \Omega .
$$

Set $\alpha(x)=\varepsilon \Phi_{1}(x), x \in \bar{\Omega}$ and

$$
H(x)=\frac{1}{a_{0}} \int_{\Omega}\left|G_{x}(x, y)\right| \sup _{u \in[\alpha(y), \beta(y)]}\left|u^{q}-u^{p+1}\right| d y, \quad x \in \bar{\Omega},
$$

which together with (4.5) and the definition of $H_{1}(x)$ implies that

$$
H(x) \leq H_{1}(x), \quad x \in \Omega .
$$

Then

$$
b_{0}=a\left(\int_{\Omega} H^{2}(x) d x\right) \leq b_{1}, \text { i.e., } \quad \frac{1}{b_{0}} \geq \frac{1}{b_{1}} .
$$

It follows from (4.3)-(4.5) that

$$
\begin{aligned}
& \left\{\begin{array}{l}
-\Delta \alpha(x)=\varepsilon \lambda \Phi_{1}(x) \\
\quad \leq \frac{\lambda}{b_{0}} \alpha^{q}(x)-\frac{1}{a_{0}} \alpha(x)^{p+1}, \quad x \text { in } \Omega, \\
\alpha(x)>0, \quad x \text { in } \Omega \\
\alpha(x)=0, \quad x \text { on } \partial \Omega
\end{array}\right. \\
& \left\{\begin{aligned}
-\Delta \beta(x) & =k_{2}>\frac{1}{a_{0}} k_{2}^{q} \\
& \geq \frac{\lambda}{a_{0}} \beta^{q}(x)-\frac{1}{b_{0}} \beta(x)^{p+1}, \quad x \text { in } \Omega, \\
\beta(x)>0, & x \text { in } \Omega, \\
\beta(x)=0, & x \text { on } \partial \Omega
\end{aligned}\right.
\end{aligned}
$$

and

$$
\alpha(x) \leq \beta(x), \quad x \in \bar{\Omega} .
$$

Now Theorem 2.2 guarantees that $(4.1)_{\lambda}$ has at least one positive solution $u_{\lambda}$ with

$$
\alpha(x) \leq u_{\lambda}(x) \leq \beta(x), \quad x \in \bar{\Omega}, \quad \lambda \in(0,1] .
$$

In the following we consider $C=\left\{\left(\lambda, u_{\lambda}\right) \mid \lambda>0, u_{\lambda}\right.$ is a positive solution to $\left.(4.1)_{\lambda}\right\}$. Obviously, $C$ is not empty. From Theorem 3.8 in [34], $C$ is unbounded. 
Moreover, for $\left(\lambda, u_{\lambda}\right) \in C$, one has

$$
\left\{\begin{aligned}
-\Delta u_{\lambda} & =\frac{1}{a\left(\int_{\Omega}\left|\nabla u_{\lambda}(x)\right|^{2}\right) d x} \lambda u_{\lambda}^{q}(x)-u_{\lambda}^{p+1}(x) \\
& \leq \frac{\lambda}{a_{0}} u_{\lambda}^{q}(x), x \in \Omega \\
u_{\lambda}(x) & =0, \quad x \text { on } \partial \Omega .
\end{aligned}\right.
$$

Since equation

$$
\left\{\begin{array}{l}
-\Delta u(x)=\frac{\lambda}{a_{0}} u^{q}(x), x \in \Omega, \\
u(x)=0, \quad x \text { on } \partial \Omega
\end{array}\right.
$$

has a unique positive solution $v_{\lambda}$ for all $\lambda>0$ and $u_{\lambda}$ is a sub-solution to (4.6), one has

$$
u_{\lambda}(x) \leq v_{\lambda}(x), \quad x \in \bar{\Omega},
$$

which together with the unboundedness of $C$ implies that $(4.1)_{\lambda}$ has at least one positive solutions for all $\lambda>0$. The proof is complete.

Now we consider the problem $(4.2)_{\lambda}$. In [13], the authors discussed the following problems

$$
\left\{\begin{array}{l}
-\frac{1}{\lambda} \Delta u(x)=m u(x)-u(x)^{p+1}, \quad x \text { in } \Omega \\
u>0, \quad x \text { in } \Omega \\
u=0, \quad x \text { on } \partial \Omega
\end{array}\right.
$$

where $\Omega \subseteq R^{N}$ is a smooth bounded domain and $m>0$ and obtained the following Lemma.

Lemma 4.1. ( see [13]) For $\lambda>\lambda_{1},(4.7)_{\lambda}$ has a unique positive solution $\theta_{\lambda, m}$ and for any compact $K \subseteq \Omega$,

$$
\lim _{\lambda \rightarrow+\infty} \theta_{\lambda, m}(x)=m^{1 / p}
$$

uniformly on $K$.

From the ideas in [5] and [13], we have the following result.

Theorem 4.2. Suppose the following conditions are satisfied:

(1)

$$
\lim _{u \rightarrow+\infty} \frac{u}{a\left(u^{2(p+1)} c\right)}=+\infty, \quad \forall c>0
$$

$$
\varliminf_{u \rightarrow+\infty} \frac{u^{p}}{u^{2}+f(u)}>0 .
$$

Then 
(1) if $\lambda<-g_{\infty},(4.2)_{\lambda}$ has no positive solution;

(2) there exists a $\lambda_{0}>0$ such that $(4.2)_{\lambda}$ has at least one positive solution $u_{\lambda}$ for all $\lambda \geq \lambda_{0}$; and moreover if $a(t)$ is bounded, for any compact $K \subseteq \Omega$,

$$
\lim _{\lambda \rightarrow+\infty} \frac{u_{\lambda}}{\lambda^{1 / p}}=1
$$

uniformly on $K$.

Proof. (1) Suppose $\lambda<-g_{\lambda}$ and $u_{\lambda}$ is a positive solution with $u_{\lambda}\left(x_{0}\right)=$ $\max _{x \in \bar{\Omega}} u_{\lambda}(x), x_{0} \in \Omega$. Then

$$
0 \leq-\Delta u_{\lambda}\left(x_{0}\right)=\frac{1}{a\left(\int_{\Omega}\left|\nabla u_{\lambda}(x)\right|^{2} d x\right)} u_{\lambda}\left(x_{0}\right)\left[\lambda+g\left(u_{\lambda}\left(x_{0}\right)\right)\right],
$$

which means that

$$
\lambda \geq-g\left(u_{\lambda}\left(x_{0}\right)\right) \geq-g_{\infty} .
$$

This is a contradiction. Then $(4.2)_{\lambda}$ has no positive solution if $\lambda<-g_{\infty}$.

(2) It is easy to see that from (4.9), $p \geq 2$ and

$$
\varliminf_{u \rightarrow+\infty} \frac{u^{p}}{f(u)}>0
$$

which implies that there is a $c_{0}>0$ and $c_{1}>0$ such that

$$
f(u) \leq c_{0} u^{p}, \quad \forall u \geq c_{1} .
$$

Let $K_{0}=\max _{u \in\left[0, c_{1}\right]} f(u)+1$. One has

$$
f(u) \leq K_{0}+c_{0} u^{p}, \quad \forall u \geq 0
$$

and then

$$
\lambda u+f(u) \leq \lambda u+K_{0}+c_{0} u^{p}, \quad \forall u \geq 0 .
$$

Choose $K_{0}^{\prime}>0$ big enough such that

$$
\frac{\lambda^{p}}{\lambda^{2}+K_{0}+c_{0} \lambda^{p}} \geq \frac{1}{c_{0}+2}, \quad \forall \lambda \geq K_{0}^{\prime}
$$

It follows from (4.8) and (4.10) that there is a $\lambda_{0}>\max \left\{1, c_{1}, K_{0}^{\prime}\right\}$ big enough such that

$\frac{\lambda}{a\left(\lambda^{2(p+1)}\left(c_{0}+3\right)^{2} \frac{1}{a_{0}^{2}} \int_{\Omega}\left|\int_{\Omega}\right| G_{x}(x, y)|d y|^{2} d x\right)} \frac{\lambda^{p}}{\lambda^{2}+K_{0}+c_{0} \lambda^{p}}>\frac{1}{a_{0}}, \quad \forall \lambda \geq \lambda_{0}$ 
and

$$
\frac{\lambda}{a\left(\lambda^{2(p+1)}\left(c_{0}+3\right)^{2} \frac{1}{a_{0}^{2}} \int_{\Omega}\left|\int_{\Omega}\right| G_{x}(x, y)|d y|^{2} d x\right)}>\lambda_{1}, \quad \forall \lambda \geq \lambda_{0},
$$

where $\lambda_{1}$ is the principle eigenvalue of problem (3.3).

For $\lambda \geq \lambda_{0}$, let $\beta(x) \equiv \lambda, \forall x \in \bar{\Omega}$ and

$$
b_{1}=a\left(\lambda^{2(p+1)}\left(c_{0}+3\right)^{2} \frac{1}{a_{0}^{2}} \int_{\Omega}\left|\int_{\Omega}\right| G_{x}(x, y)|d y|^{2} d x\right) .
$$

It follows from (4.12) that $\frac{\lambda}{b_{1}}>\lambda_{1}$ for all $\lambda \geq \lambda_{0}$. Since $p \geq 2$, choose $1>\varepsilon>0$ small enough such that

$$
\varepsilon \Phi_{1}(x)<\lambda=\beta(x), \quad \forall x \in \bar{\Omega}
$$

and

$$
\lambda_{1} \leq \frac{\lambda}{b_{1}}-\frac{1}{a_{0}} \varepsilon^{p} \Phi_{1}^{p}(x), \quad \forall x \in \bar{\Omega},
$$

which guarantees that

$$
\lambda_{1} \varepsilon \Phi_{1}(x) \leq \frac{\lambda}{b_{1}} \varepsilon \Phi_{1}(x)-\frac{1}{a_{0}}\left(\varepsilon \Phi_{1}(x)\right)^{p+1}, \quad \forall x \in \bar{\Omega} .
$$

Set $\alpha(x)=\varepsilon \Phi_{1}(x), x \in \bar{\Omega}$ and

$$
H(x)=\frac{1}{a_{0}} \int_{\Omega}\left|G_{x}(x, y)\right| \sup _{u \in[\alpha(y), \beta(y)]}\left|\lambda u+f(u)-u^{p+1}\right| d y, \quad x \in \bar{\Omega} .
$$

From the definition of $\lambda_{0}$, one has

$$
H(x) \leq \frac{1}{a_{0}} \int_{\Omega}\left|G_{x}(x, y)\right| d y\left(c_{0}+3\right) \lambda^{p+1}, \quad x \in \bar{\Omega} .
$$

Then

$$
b_{0}=a\left(\int_{\Omega} H^{2}(x) d x\right) \leq a\left(\lambda^{2(p+1)}\left(c_{0}+3\right)^{2} \frac{1}{a_{0}^{2}} \int_{\Omega}\left|\int_{\Omega}\right| G_{x}(x, y)|d y|^{2} d x\right)=b_{1},
$$

i.e.,

$$
\frac{1}{b_{0}} \geq \frac{1}{b_{1}}
$$

From (4.11) and (4.15), one has

$$
\frac{\lambda}{b_{0}} \frac{\lambda^{p}}{\lambda^{2}+f(\lambda)} \geq \frac{\lambda}{b_{1}} \frac{\lambda^{p}}{\lambda^{2}+K_{0}+c_{0} \lambda^{p}}>\frac{1}{a_{0}}, \quad \forall \lambda \geq \lambda_{0}
$$


i.e.

$$
0>\frac{1}{a_{0}}\left(\lambda^{2}+f(\lambda)\right)-\frac{1}{b_{0}} \lambda^{p+1}, \quad \forall \lambda \geq \lambda_{0} .
$$

It follows from (4.13)-(4.16) that

$$
\left\{\begin{aligned}
-\Delta \alpha(x) & =\lambda_{1} \varepsilon \Phi_{1}(x) \\
& \leq \frac{\lambda}{b_{1}} \varepsilon \Phi_{1}(x)-\frac{1}{a_{0}}\left(\varepsilon \Phi_{1}(x)\right)^{p+1} \\
& \leq \frac{1}{b_{1}}\left[\lambda \varepsilon \Phi_{1}(x)+f\left(\varepsilon \Phi_{1}(x)\right)\right]-\frac{1}{a_{0}}\left(\varepsilon \Phi_{1}(x)\right)^{p+1} \\
& \leq \frac{1}{b_{0}}[\lambda \alpha(x)+f(\alpha(x))]-\frac{1}{a_{0}}(\alpha(x))^{p+1} \\
\alpha(x)>0, & x \text { in } \Omega \\
\alpha(x)=0, & x \text { on } \partial \Omega
\end{aligned}\right.
$$

and

$$
\left\{\begin{aligned}
-\Delta \beta(x) & =0 \\
& >\frac{1}{a_{0}}(\lambda \beta(x)+f(\beta(x)))-\frac{1}{b_{0}} \beta(x)^{p+1}, \quad x \text { in } \Omega \\
\beta(x)>0, & x \text { in } \Omega \\
\beta(x)=0, & x \text { on } \partial \Omega
\end{aligned}\right.
$$

with

$$
\alpha(x) \leq \beta(x), \quad x \in \bar{\Omega} .
$$

Now Theorem 2.2 guarantees that for $\lambda \geq \lambda_{0},(4.2)_{\lambda}$ has at least one positive solution $u_{\lambda}$ with

$$
\alpha(x) \leq u_{\lambda}(x) \leq \beta(x), \quad x \in \bar{\Omega}, \quad \forall \lambda \geq \lambda_{0} .
$$

Suppose that $u_{\lambda}$ is a positive solution of (4.2) for $\lambda \geq \lambda_{0}$. We show that for any $\varepsilon>0$, there is a $\lambda(\varepsilon)>\lambda_{0}$ such that

$$
\left|\frac{1}{\lambda} \frac{f\left(u_{\lambda}\right)}{u_{\lambda}}\right|<\varepsilon, \quad \forall \lambda>\lambda(\varepsilon) .
$$

Let $c_{\lambda}$ be the largest real number such that

$$
\lambda+g\left(c_{\lambda}\right)=0 .
$$

Observe $\lim _{\lambda \rightarrow+\infty} c_{\lambda}=+\infty$. Moreover, $\lambda+g(u)<0$ for all $u>c_{\lambda}$. For any positive solution $u_{\lambda}$, we have $u_{\lambda}\left(x_{0}\right) \leq c_{\lambda}$, where $u_{\lambda}\left(x_{0}\right)=\max _{x \in \bar{\Omega}} u_{\lambda}(x)$. Hence, $u_{\lambda}(x) \leq c_{\lambda}$ for all $x \in \bar{\Omega}$. Since

$$
\frac{\lambda^{1 / p}}{c_{\lambda}}=\left[1-\frac{f\left(c_{\lambda}\right)}{c_{\lambda}^{p+1}}\right]^{1 / p} \text { and } \lim _{u \rightarrow+\infty} \frac{f(u)}{u^{p+1}}=0
$$


one has

$$
\lim _{\lambda \rightarrow+\infty} \frac{\lambda^{1 / p}}{c_{\lambda}}=1
$$

For given $\varepsilon>0$, as $\lim _{u \rightarrow+\infty} \frac{f(u)}{u^{p+1}}=0$, there is a $M_{\varepsilon}>0$ such that

$$
\left|\frac{f(u)}{u^{p+1}}\right| \leq \frac{\varepsilon}{3}, \quad \forall u \geq M_{\varepsilon}
$$

From (4.18), there is a $\hat{\lambda}>0$ such that

$$
\frac{c_{\lambda}^{p}}{\lambda}<\frac{3}{2}, \quad \forall \lambda \geq \hat{\lambda}
$$

Let

$$
\lambda(\varepsilon)=\max \left\{\lambda_{0}+1, \hat{\lambda}, \frac{1}{\varepsilon} \sup _{u \in\left[0, M_{\varepsilon}\right]}\left|\frac{f(u)}{u}\right|\right\} .
$$

For all $\lambda>\lambda(\varepsilon)$ and $u_{\lambda}$ a solution, then if $u_{\lambda}(x) \geq M_{\varepsilon}$, one has

$$
\left|\frac{1}{\lambda} \frac{f\left(u_{\lambda}(x)\right)}{u_{\lambda}(x)}\right|=\left|\left(\frac{u_{\lambda}(x)}{c_{\lambda}}\right)^{p} \frac{c_{\lambda}^{p}}{\lambda} \frac{f\left(u_{\lambda}(x)\right)}{u_{\lambda}^{p+1}(x)}\right| \leq \frac{3}{2} \frac{\varepsilon}{3}=\frac{\varepsilon}{2} ;
$$

if $u_{\lambda}(x) \leq M_{\varepsilon}$, one has

$$
\left|\frac{1}{\varepsilon} \frac{f\left(u_{\lambda}(x)\right)}{u_{\lambda}(x)}\right|=\left|\frac{1}{\varepsilon} \sup _{u \in\left[0, M_{\varepsilon}\right]} \frac{f(u)}{u}\right|<\lambda(\varepsilon)<\lambda
$$

i.e.,

$$
\left|\frac{1}{\lambda} \frac{f\left(u_{\lambda}(x)\right)}{u_{\lambda}(x)}\right|<\varepsilon .
$$

Combining (4.19) and (4.20), we get (4.17).

We suppose that $a(t)$ is bounded, i.e., there is a $M_{0}>0$ such that $a_{0} \leq$ $a(t) \leq M_{0}$. Let $\rho(\lambda)=a\left(\int_{\Omega}\left|\nabla u_{\lambda}(x)\right|^{2} d x\right)$. Obviously, $a_{0} \leq \rho(\lambda) \leq M_{0}$ for $\lambda>\lambda_{0}$. Let $v_{\lambda}=\lambda^{-1 / p} u_{\lambda}$. It is easy to see that $v_{\lambda}$ satisfies

$$
\left\{\begin{array}{l}
-\frac{\rho(\lambda)}{\lambda} \Delta v_{\lambda}=-\frac{1}{\lambda / \rho(\lambda)} \Delta v_{\lambda}=v_{\lambda}+\frac{f\left(\lambda^{1 / p} v_{\lambda}\right)}{\lambda^{1+(1 / p)}}-v_{\lambda}^{p+1}, \quad x \in \Omega \\
\left.v_{\lambda}\right|_{\partial \Omega}=0
\end{array}\right.
$$

and

$$
\lim _{\lambda \rightarrow+\infty} \frac{\lambda}{\rho(\lambda)}=+\infty
$$


Now we consider

$$
\left\{\begin{array}{l}
-\frac{\rho(\lambda)}{\lambda} \Delta v=m v-v^{p+1}, \quad x \in \Omega \\
\left.v\right|_{\partial \Omega}=0 .
\end{array}\right.
$$

Let $\theta_{\lambda, \rho(\lambda), m}$ be a positive solution.

Now we show that (for $\varepsilon>0$ small)

$$
\theta_{\lambda, \rho(\lambda), 1-\varepsilon}(x) \leq v_{\lambda}(x) \leq \theta_{\lambda, \rho(\lambda), 1+\varepsilon}(x) .
$$

For fixed $\varepsilon>0$ small, we first claim

$$
\theta_{\lambda, \rho(\lambda), 1-\varepsilon}(x) \leq v_{\lambda}(x)
$$

Without loss of generality assume $\theta_{\lambda, \rho(\lambda), 1-\varepsilon}(x) \neq v_{\lambda}(x)$, and we have

$$
\frac{v_{\lambda}^{p+1}(x)-\theta_{\lambda, \rho(\lambda), 1-\varepsilon}(x)^{p+1}}{v_{\lambda}(x)-\theta_{\lambda, \rho(\lambda), 1-\varepsilon}(x)}=\theta_{\lambda, \rho(\lambda), 1-\varepsilon}^{p}(x)+Q(x)
$$

where $Q(x)>0$ and hence

$$
\begin{gathered}
\sigma_{1}\left[-\frac{\rho(\lambda)}{\lambda} \Delta-1+\varepsilon+\frac{v_{\lambda}^{p+1}(x)-\theta_{\lambda, \rho(\lambda), 1-\varepsilon}(x)^{p+1}}{v_{\lambda}(x)-\theta_{\lambda, \rho(\lambda), 1-\varepsilon}(x)}\right] \\
>\sigma_{1}\left[-\frac{\rho(\lambda)}{\lambda} \Delta-1+\varepsilon+\theta_{\lambda, \rho(\lambda), 1-\varepsilon}(x)^{p}\right] .
\end{gathered}
$$

Hereafter, given an elliptic operator $L, \sigma_{1}(L)$ stands the principal eigenvalue of $L$ subject to the homogeneous Dirichlet boundary conditions. From the Krein-Rutmann's Theorem and the definition of $\theta_{\lambda, \rho(\lambda), 1-\varepsilon}(x)$, we have

$$
\sigma_{1}\left[-\frac{\rho(\lambda)}{\lambda} \Delta-1+\varepsilon+\theta_{\lambda, \rho(\lambda), 1-\varepsilon}(x)^{p}\right]=0 .
$$

Thus

$$
\sigma_{1}\left[-\frac{\rho(\lambda)}{\lambda} \Delta-1+\varepsilon+\frac{v_{\lambda}^{p+1}(x)-\theta_{\lambda, \rho(\lambda), 1-\varepsilon}(x)^{p+1}}{v_{\lambda}(x)-\theta_{\lambda, \rho(\lambda), 1-\varepsilon}(x)}\right]>0 .
$$

On the other hand, after some straight forward manipulations, it follows from (4.21) and (4.22) that

$$
\begin{aligned}
& {\left[-\frac{\rho(\lambda)}{\lambda} \Delta-1+\varepsilon+\frac{v_{\lambda}^{p+1}(x)-\theta_{\lambda, \rho(\lambda), 1-\varepsilon}(x)^{p+1}}{v_{\lambda}(x)-\theta_{\lambda, \rho(\lambda), 1-\varepsilon}(x)}\right]\left(v_{\lambda}(x)-\theta_{\lambda, \rho(\lambda), 1-\varepsilon}(x)\right)} \\
& =\left[\varepsilon+\frac{1}{\lambda} \frac{f\left(\lambda^{1 / p} v\right)}{\lambda^{\lambda^{1 / p}} v}\right] v_{\lambda} .
\end{aligned}
$$


From (4.17), we have

$$
\left[\varepsilon+\frac{1}{\lambda} \frac{f\left(\lambda^{1 / p} v\right)}{\lambda^{\lambda^{1 / p}} v}\right]>0
$$

for $\lambda>\lambda(\varepsilon)$. Applying the maximum principle to (4.24), we have

$$
\theta_{\lambda, \rho(\lambda), 1-\varepsilon}(x) \leq v_{\lambda}(x), \quad \forall x \in \bar{\Omega}, \quad \forall \lambda>\lambda(\varepsilon),
$$

so our claim is true.

For fixed $\varepsilon>0$ small, we next claim

$$
\theta_{\lambda, \rho(\lambda), 1+\varepsilon}(x) \geq v_{\lambda}(x)
$$

Without loss of generality assume $\theta_{\lambda, \rho(\lambda), 1+\varepsilon}(x) \neq v_{\lambda}(x)$, and we have

$$
\frac{v_{\lambda}^{p+1}(x)-\theta_{\lambda, \rho(\lambda), 1+\varepsilon}(x)^{p+1}}{v_{\lambda}(x)-\theta_{\lambda, \rho(\lambda), 1+\varepsilon}(x)}=\theta_{\lambda, \rho(\lambda), 1+\varepsilon}^{p}(x)+Q_{1}(x)
$$

where $Q_{1}(x)>0$ and hence

$$
\begin{aligned}
& \sigma_{1}\left[-\frac{\rho(\lambda)}{\lambda} \Delta-1-\varepsilon+\frac{v_{\lambda}^{p+1}(x)-\theta_{\lambda, \rho(\lambda), 1+\varepsilon}(x)^{p+1}}{v_{\lambda}(x)-\theta_{\lambda, \rho(\lambda), 1+\varepsilon}(x)}\right] \\
& >\sigma_{1}\left[-\frac{\rho(\lambda)}{\lambda} \Delta-1-\varepsilon+\theta_{\lambda, \rho(\lambda), 1+\varepsilon}(x)^{p}\right] .
\end{aligned}
$$

From the Krein-Rutmann's Theorem and the definition of $\theta_{\lambda, \rho(\lambda), 1+\varepsilon}(x)$, we have

$$
\sigma_{1}\left[-\frac{\rho(\lambda)}{\lambda} \Delta-1-\varepsilon+\theta_{\lambda, \rho(\lambda), 1+\varepsilon}(x)^{p}\right]=0 .
$$

Thus

$$
\sigma_{1}\left[-\frac{\rho(\lambda)}{\lambda} \Delta-1-\varepsilon+\frac{v_{\lambda}^{p+1}(x)-\theta_{\lambda, \rho(\lambda), 1+\varepsilon}(x)^{p+1}}{v_{\lambda}(x)-\theta_{\lambda, \rho(\lambda), 1+\varepsilon}(x)}\right]>0 .
$$

On the other hand, after some straight forward manipulations, it follows from (4.21) and (4.22) that

$$
\begin{aligned}
& {\left[-\frac{\rho(\lambda)}{\lambda} \Delta-1-\varepsilon+\frac{v_{\lambda}^{p+1}(x)-\theta_{\lambda, \rho(\lambda), 1+\varepsilon}(x)^{p+1}}{v_{\lambda}(x)-\theta_{\lambda, \rho(\lambda), 1+\varepsilon}(x)}\right]\left(v_{\lambda}(x)-\theta_{\lambda, \rho(\lambda), 1+\varepsilon}(x)\right)} \\
& =\left[\varepsilon-\frac{1}{\lambda} \frac{f\left(\lambda^{1 / p} v\right)}{\lambda^{\lambda^{1 / p}} v}\right] v_{\lambda} .
\end{aligned}
$$


From (4.17), we have

$$
\left[\varepsilon-\frac{1}{\lambda} \frac{f\left(\lambda^{1 / p} v\right)}{\lambda^{\lambda^{1 / p}} v}\right]>0
$$

for $\lambda>\lambda(\varepsilon)$. Applying the maximum principle to (4.25), we have

$$
\theta_{\lambda, \rho(\lambda), 1+\varepsilon}(x) \geq v_{\lambda}(x)
$$

so our claim is true.

Then (4.23) is true. Next note Lemma 4.1 and the fact that $\lim _{\lambda \rightarrow+\infty} \frac{\lambda}{\rho(\lambda)}=$ $+\infty$. The proof is complete.

\section{Sufficient and necessary conditions for the existence of positive solutions when $N=1$}

In this section, we consider the problem (1.1) for the case $N=1$. First we consider

$$
\left\{\begin{array}{l}
-u^{\prime \prime}=\frac{1}{a\left(\int_{\Omega}\left|u^{\prime}\right|^{2} d x\right)} f(x, u), \quad x \in(0,1), \\
u(0)=u(1)=0 .
\end{array}\right.
$$

Now the following condition is listed for convenience:

$\left(\mathrm{H}_{1}\right) f:(0,1) \times[0,+\infty) \rightarrow[0,+\infty)$, continuous and $\exists \lambda, \mu, \delta,(0<\lambda<$ $\mu<1,0<\delta \leq 1), \forall x \in(0,1), v \in(0,+\infty)$, we have

$$
\begin{gathered}
c_{0}^{\mu} f(x, v) \leq f\left(x, c_{0} v\right) \leq c_{0}^{\lambda} f(x, v), \quad 0 \leq c_{0} \leq \delta, \\
c_{0}^{\lambda} f(x, v) \leq f\left(x, c_{0} v\right) \leq c_{0}^{\mu} f(x, v), \quad c_{0} \geq 1 / \delta .
\end{gathered}
$$

Lemma 5.1.(see [1]) Suppose $u \geq 0$ is concave on $[0,1]$ with $u(0)=u(1)=0$. Then

$$
u(x) \geq|u|_{\infty} x(1-x), \quad t \in[0,1]
$$

Using a standard idea (see for example [33]) sufficient and necessary conditions for the existence of positive solutions to (5.1) are obtained.

Theorem 5.1 Suppose $\left(H_{1}\right)$ holds. Then a necessary and sufficient condition for positive solutions $C^{1}[0,1]$ of $(5.1)$ is

$$
0<\int_{0}^{1} f(x, x(1-x)) d x<\infty .
$$


Proof. Necessity. Suppose that $u_{0}$ is a positive solution with $u_{0} \in C^{1}[0,1]$. It is easy to see that $u_{0}^{\prime}(0)>0$ and $u_{0}^{\prime}(1)<0$. Lemma 5.1 implies that there is a $k_{0}>1 / \delta$ big enough such that

$$
\frac{x(1-x)}{k_{0} u_{0}(x)} \leq \delta, \quad x \in(0,1),
$$

which together with (5.2) and (5.3) implies

$$
\begin{aligned}
f(x, x(1-x)) & =f\left(x, \frac{x(1-x)}{k_{0} u_{0}(x)} k_{0} u_{0}(x)\right) \\
& \leq\left(\frac{x(1-x)}{k_{0} u_{0}(x)}\right)^{\lambda} f\left(x, k_{0} u_{0}(x)\right) \\
& \leq\left(\frac{x(1-x)}{k_{0} u_{0}(x)}\right)^{\lambda} k_{0}^{\mu} f\left(x, u_{0}(x)\right) \\
& \leq \delta^{\lambda} k_{0}^{\mu} f\left(x, u_{0}(x)\right), \quad x \in(0,1) .
\end{aligned}
$$

Choose $x_{0} \in(0,1)$ with $u_{0}\left(x_{0}\right)=\max _{x \in[0,1]} u_{0}(x)$. It is easy to see that $u_{0}^{\prime}\left(x_{0}\right)=0$ with $u_{0}^{\prime}(x)>0$ for $x \in\left[0, x_{0}\right)$ and $u_{0}^{\prime}(x)<0$ for $x \in\left(x_{0}, 1\right]$. Moreover, it follows from (5.5) that

$$
\begin{aligned}
u_{0}^{\prime}(0) & =u_{0}^{\prime}(0)-u_{0}^{\prime}\left(x_{0}\right) \\
& =\frac{1}{a\left(\int_{0}^{1}\left|u_{0}^{\prime}(x)\right|^{2} d x\right)} \int_{0}^{x_{0}} f\left(s, u_{0}(s)\right) d s \\
& \geq \delta^{-\lambda} k_{0}^{-\mu} \frac{1}{a\left(\int_{0}^{1}\left|u_{0}^{\prime}(x)\right|^{2} d x\right)} \int_{0}^{x_{0}} f(s, s(1-s)) d s .
\end{aligned}
$$

A similar argument shows that

$$
-u_{0}^{\prime}(1) \geq \delta^{-\lambda} k_{0}^{-\mu} \frac{1}{a\left(\int_{0}^{1}\left|u_{0}^{\prime}(x)\right|^{2} d x\right)} \int_{x_{0}}^{1} f(s, s(1-s)) d s .
$$

We deduce from (5.6) and (5.7) that

$$
\int_{0}^{1} f(x, x(1-x)) d x<+\infty
$$

Sufficiency. Let

$$
h(x)=(1-x) \int_{0}^{x} s f(s, s(1-s)) d s+x \int_{x}^{1}(1-s) f(s, s(1-s)) d s, \quad \forall x \in[0,1] .
$$

From Lemma 5.1, we can see $h(x) \geq x(1-x)|h|_{\infty}$. It follows from (5.4) that $h \in C^{1}([0,1])$, which implies that there exists a $a_{2}>0$ such that

$$
h(x) \leq a_{2} x(1-x), \quad x \in[0,1] .
$$


Let $k_{2}>0$ be big enough such that

$$
\frac{1}{a_{0}} a_{2}^{\mu} k_{2}^{\mu-1} \delta^{\lambda-\mu} \leq 1, \quad|h|_{\infty} k_{2}>1 .
$$

Define $\beta(x)=k_{2} h(x), x \in[0,1]$. From (5.2) and (5.3) we have

$$
\begin{aligned}
\frac{1}{a_{0}} f(x, \beta(x)) & =\frac{1}{a_{0}} f\left(x, k_{2} h(x)\right) \\
& =\frac{1}{a_{0}} f\left(x, \frac{k_{2} h(x)}{\delta x(1-x)} \delta x(1-x)\right) \\
& \leq \frac{1}{a_{0}}\left(\frac{k_{2} h(x)}{\delta x(1-x)}\right)^{\mu} \delta^{\lambda} f(x, x(1-x)) \\
& \leq \frac{1}{a_{0}} a_{2}^{\mu} k_{2}^{\mu-1} \delta^{\lambda-\mu} k_{2} f(x, x(1-x)) \\
& \leq k_{2} f(x, x(1-x)), \quad x \in(0,1) .
\end{aligned}
$$

Let

$$
\Gamma_{1}(x)=\frac{1}{a_{0}} \int_{0}^{1}\left|G_{x}(x, s)\right| \sup _{r \in\left[0, k_{2} h(s)\right]} f(s, r) d s, \quad x \in[0,1],
$$

where $G(x, s)$ is the Green's function for $-u^{\prime \prime}(x)=h$ with $u(0)=u(1)=0$ and

$$
b_{1}=a\left(\int_{0}^{1} \Gamma_{1}^{2}(x) d x\right) .
$$

Now choose $k_{1}<k_{2}$ small enough such that

$$
k_{1}^{\mu-1} \delta^{\lambda-\mu}|h|_{\infty}^{\mu} \frac{1}{b_{1}} \geq 1, \quad k_{1} a_{2} \leq \delta^{2}, \quad k_{1} h(x) \leq 1 .
$$

Let $\alpha(x)=k_{1} h(x)$,

$$
\Gamma(x)=\frac{1}{a_{0}} \int_{0}^{1}\left|G_{x}(x, s)\right| \sup _{r \in\left[k_{1} h(s), k_{2} h(s)\right]} f(s, r) d s, \quad x \in[0,1],
$$

and

$$
b_{0}=a\left(\int_{0}^{1} \Gamma^{2}(x) d x\right) .
$$

It is easy to see that

$$
b_{0} \leq b_{1} \text {, i.e., } \frac{1}{b_{0}} \geq \frac{1}{b_{1}} .
$$


It follows from (5.2) and (5.3) that

$$
\begin{aligned}
\frac{1}{b_{0}} f(x, \alpha(x)) & \geq \frac{1}{b_{1}} f\left(x, k_{1} h(x)\right) \\
& =\frac{1}{b_{1}} f\left(x, \frac{k_{1} h(x)}{\delta x(1-x)} \delta x(1-x)\right) \\
& \geq \frac{1}{b_{1}}\left(\frac{k_{1} h(x)}{\delta x(1-x)}\right)^{\mu} \delta^{\lambda} f(x, x(1-x)) \\
& \geq \frac{1}{b_{1}}|h|_{\infty}^{\mu} k_{1}^{\mu-1} \delta^{\lambda-\mu} k_{1} f(x, x(1-x)) \\
& \geq k_{1} f(x, x(1-x)), \quad x \in(0,1) .
\end{aligned}
$$

Consequently, (5.8) and (5.9) guarantee that

$$
\left\{\begin{aligned}
-\beta^{\prime \prime}(x) & =-\left(k_{2} h(x)\right)^{\prime \prime} \\
& =k_{2} f(x, x(1-x)) \\
& \geq \frac{1}{a_{0}} f(x, \beta(x)), \\
\beta(0) & =\beta(0)=0,
\end{aligned}\right.
$$

and

$$
\left\{\begin{aligned}
-\alpha^{\prime \prime}(x) & =-\left(k_{1} h(x)\right)^{\prime \prime} \\
& =k_{1} f(x, x(1-x)) \\
& \leq \frac{1}{b_{0}} f(x, \alpha(x)) \\
\alpha(0) & =\alpha(0)=0 .
\end{aligned}\right.
$$

Moreover, for $\alpha(x) \leq u \leq \beta(x)$, choose $c>0$ big enough such that

$$
\frac{c u}{k_{2} x(1-x)} \geq \frac{1}{\delta}, \quad \text { and } \frac{k_{2}}{c} \leq \delta, \quad x \in(0,1),
$$

and from (5.2) and (5.3), we have

$$
\begin{aligned}
0 & \leq f(x, u) \\
& =f\left(x, \frac{k_{2}}{c} \frac{c u}{k_{2} x(1-x)} x(1-x)\right) \\
& \left.\leq\left(\frac{k_{2}}{c}\right)^{\lambda}\left(\frac{c u}{k_{2} x(1-x)}\right)^{\mu} f(x, x(1-x))\right) \\
& \left.\leq\left(\frac{k_{2}}{c}\right)^{\lambda}\left(c a_{2}\right)^{\mu} f(x, x(1-x))\right),
\end{aligned}
$$

which together (5.4) guarantees that

$$
\int_{0}^{1}|f(x, u)| d x \leq\left(\frac{k_{2}}{c}\right)^{\lambda}\left(c a_{2}\right)^{\mu} \int_{0}^{1} f(x, x(1-x)) d x<+\infty, \quad \forall \alpha(x) \leq u \leq \beta(x) .
$$


From Theorem 2.1, (5.1) has at least one positive solution $u \in C^{1}[0,1]$ with $\alpha(x) \leq u(x) \leq \beta(x), x \in[0,1]$. The proof is complete.

\section{References}

[1] R. P. Agarwal and D. O'Regan, A survey of recent results for initial and boundary value problems singular in the dependent variable. Original Research Article Handbook of Differential Equations: Ordinary Differential Equations, 1, 2000, 1-68.

[2] C. O. Alves, D. P. Covei, Existence of solution for a class of nonlocal elliptic problem via sub-supersolution method, Nonlinear Analysis: Real World Applications, 23, 2015, 1-8.

[3] C. O. Alves, F. J. S. A.Corrêa, A sub-supersolution approach for a quasilinear Kirchhoff equation, Journal of Mathematical Physics, 56, 051501, 2015.

[4] C. O. Alves, F. J. S. A. Corrêa, T. F. Ma, Positive solutions for a quasilinear elliptic equation of kirchhoff-type, Computers and Mathematics with Applications, 49, 2005, 85-93.

[5] H. Berestycki, Le nombre de solutions de certains problémes semilinéaires elliptiques, J. Funct. Anal., 40, 1981, 1-29.

[6] B. Cheng, X. Wu, Existence results of positive solutions of Kirchhoff type problems, Nonlinear Anal., 71, 2009, 4883-4892.

[7] H. Cheng, R. Yuan, Existence and stability of traveling waves for Leslie-Gower predator-prey system with nonlocal diffusion, Discrete and Continuous Dynamical Systems-Series A, 37, 2017, 5433-5454.

[8] N. T. Chung, An existence result for a class of Kirchhoff type systems via sub and supersolutions method, Appl. Math. Lett., 35, 2014, 95-101.

[9] N. T. Chung, G. A. Afrouzi, Existence of positive solutions for a class of nonlocal elliptic systems with multiple parameter, Matematicki Vesnik, 67(3), 2015, 166-173.

[10] F. J. S. A.Corrêa, M. Delgado, A. Suárez, Some non-local problems with nonlinear diffusion, Mathematical and Computer Modelling, 54, 2011, 2293-2305.

[11] C. De Coster, Existence and localization of solution for second order elliptic BVP in presence of lower and upper solutions without any Order, J. Differential Equations, 145, 1998, 420-452.

[12] Y. Deng, S. Peng, W. Shuai, Existence and asymptotic behavior of nodal solutions for the Kirchhoff-type problems in, J. Functional Analysis, 269, 2015, 3500-3527.

[13] J. M. Fraile, J. López-Gómez and J. C. Delis, On the global structure 
of the set of positive solutions of some semilinear elliptic boundary value problems, J. Differential. Equations, 123, 1995, 180-212.

[14] G. M. Figueiredo, A. Suárez, Some remarks on the comparison principle in Kirchhoff equations, arXiv:1510.02151v1 [math.AP], 2015.

[15] G. M. Figueiredo, A. Suárez, The sub-supersolution method for Kirchhoff systems, Contributions to Nonlinear Elliptic Equations and Systems, Progress in Nonlinear Differential Equations and Their Applications 86, DOI 10.1007/978-3-319-19902-3 14.Springer International Publishing Switzerland 2015.

[16] S. M. Gomes, On a singular nonlinear elliptic problems, Siam J. Math. Anal., 17(6), 1986, 1359-1369.

[17] X. Han and G. Dai, On the sub-supersolution method for $p(x)$ Kirchhoff type equa- tions, Journal of Inequalities and Applications, 2012, (2012), 283.

[18] X. He, W. Zou, Infinitely many positive solutions for Kirchhoff-type problems, Nonlinear Anal., 70, 2009, 1407-1414.

[19] X. He, W. Zou, Existence and concentration behavior of positive solutions for a Kirchhoff equation in $R^{3}, J$. Differential Equations, 252(2), 2012, 1813-1834.

[20] J. Jin, X. Wu, Infinitely many radial solutions for Kirchhoff-type problems in $R^{N}$, J. Math. Anal. Appl., 368(2), 2010, 564-574. [21] G. Kirchhoff, newblock Mechanik. newblock Teubner, Leipzig, 1883.

[22] A. C. Lazer, P. J. McKenna, On a singular nonlinear elliptic boundary-value problems, Proceeding of American Mathematical Society, 111(3), 1991, 721-730.

[23] C. Lei, J. Liao, C. Tang, Multiple positive solutions for Kirchhoff type of problems with singularity and critical exponents, J. Math. Anal. Appl., 421, 2015, 521-538.

[24] Y. Li, F. Li, J. Shi, Existence of a positive solution to Kirchhoff type problems without compactness conditions, J. Differential Equations, 253(7), 2012, 2285-2294.

[25] X. Li, S. Song, Stabilization of delay systems: delay-dependent impulsive control, IEEE Transactions on Automatic Control, 62, 2017, 406-411.

[26] X. Li, J. Wu, Stability of nonlinear differential systems with statedependent delayed impulses, Automatica, 64, 2016, 63-69.

[27] Z. Liang, F. Li, J. Shi, Positive solutions to Kirchhoff type equations with nonlinearity having prescribed asymptotic behavior, Ann. I. H. Poincaré, 31, 2014, 155-167.

[28] J. Liao, P. Zhang, J. Liu, C. Tang, Existence and multiplicity of positive solutions for a class ofKirchhoff type problems with singularity, $J$. Math. Anal. Appl., 430, 2015, 1124-1148. 
[29] J. L. Lions, On some questions in boundary value problems of mathematical physics, In Proceedings of International Symposium on Continuum Mechanics and Partial Differential Equations, 36, 1977, 284-346.

[30] X. Liu, Y. Sun, Multiple positive solutions for Kirchhoof type problems with singularity, Communications on Pure and Applied Analysis, 22(2), 2013, 721-733.

[31] R. Ma, G. Dai and C. Gao, Existence and multiplicity of positive solutions for a class of $\mathrm{p}(\mathrm{x})$-Kirchhoff type equations, Boundary Value Problems, 2012, 2012, 16.

[32] T. F. Ma, J. E. M. Rivera, Positive solutions for a nonlinear nonlocal elliptic transmission problem, Appl. Math. Lett., 16, 2003, 243-248.

[33] K. Perera, Z. Zhang, Nontrivial solutions of Kirchhoff-type problems via the Yang index, J. Differential Equations, 221, 2006, 246-255.

[34] P. H. Rabinowitz, Some global results for nonlinear eigenvalue problems, Journal of Functional Analysis, 7(3),1971, 487-513.

[35] W. Shuai, Sign-changing solutions for a class of Kirchhoff-type problem in bounded domains, J. Differential Equations, 259, 2015, 1256-1274.

[36] Z. Wei, Positive solutions of nonresonant singular boundary value problems for second order differential equation system, Nagoya Math. Anal. J., 162, 2001, 127-148.

[37] B. Yan, Q. Ren, Existence, uniqueness and multiplicity of positive solutions for some nonlocal singular elliptic problems, Electronic Journal of Differential Equations, 138, 2017, 1-21.

[38] B. Yan, D. Wang, The multiplicity of positive solutions for a class of nonlocal elliptic problemJ. Math. Anal. Appl., 442, 2016, 72-102.

[39] Z. Zhang, Nonexistence of positive classical solutions of a singular nonlinear Dirichlet problem with a convection term, Nonlinear Analysis: TMA, 21, 1996, 957-961.

Baoqiang YAN,

School of Mathematical Sciences,

Shandong Normal University,

Jinan, 250014, P. R. China.

Email: yanbqcn@aliyun.com

Donal O'REGAN,

School of Mathematics, Statistics and Applied Mathematics,

National University of Ireland,

Galway, Ireland.

Email: donal.oregan@nuigalway.ie

Ravi P. AGARWAL,

Department of Mathematics,

Texas A and M University-Kingsville,

Texas 78363, USA.

Email: Ravi.Agarwal@tamuk.edu 